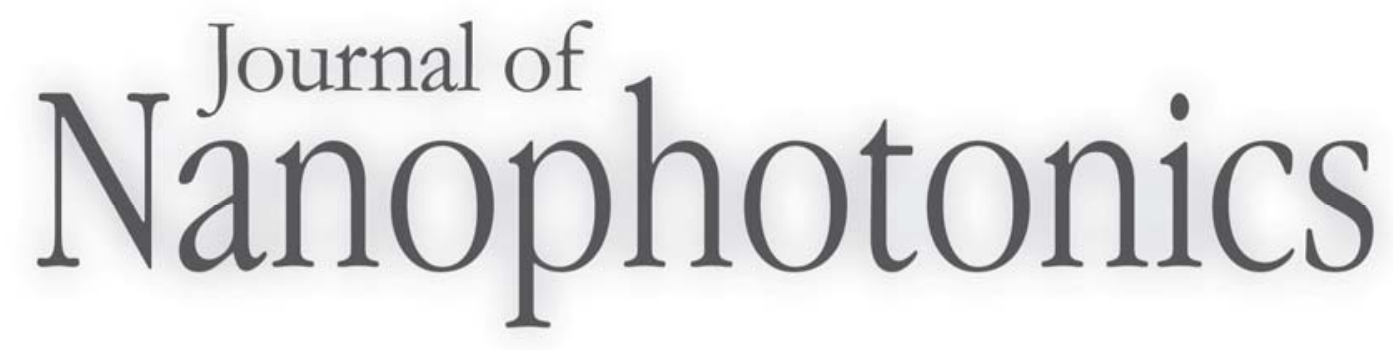

SPIEDigitalLibrary.org/jnp

\title{
Carbon nanotubes for next generation very large scale integration interconnects
}

Ashok Srivastava

Yao Xu

Ashwani K. Sharma

\section{O SPIE}




\title{
Carbon nanotubes for next generation very large scale integration interconnects
}

\author{
Ashok Srivastava, ${ }^{\mathrm{a}}$ Yao Xu, ${ }^{\mathrm{a}}$ and Ashwani K. Sharma ${ }^{\mathrm{b}}$ \\ ${ }^{a}$ Louisiana State University, Department of Electrical and Computer Engineering, \\ Baton Rouge, LA 70803-5901, U.S.A. \\ a ashok@ece.lsu.edu \\ ${ }^{\mathrm{b}}$ AFRL/RVSE, Electronics Foundations Group, \\ 3550 Aberdeen Avenue SE, Kirtland AFB, NM 87117, U.S.A.
}

\begin{abstract}
We investigated the application of one-dimensional fluid model in modeling of electron transport in carbon nanotubes and equivalent circuits for interconnections and compared the performances with the currently used copper interconnects in very-large-scale integration (VLSI) circuits. In this model, electron transport in carbon nanotubes is regarded as quasi one-dimensional fluid with strong electron-electron interaction. Verilog-AMS in Cadence/Spectre was used in simulation studies. Carbon nanotubes of the types single-walled, multiwalled and bundles were considered for ballistic transport region, local and global interconnections. Study of the S-parameters showed higher transmission efficiency and lower reflection losses. Theoretical modeling and computer-aided simulation studies through a complementary CNT-FET inverter pair, interconnected through a wire, exhibited reduced delays and power dissipations for carbon nanotube interconnects in comparison to copper interconnects in $22 \mathrm{~nm}$ and lower technology nodes. The performance of CNT interconnects was shown to be further improved with increase in number of metallic carbon nanotubes. Our study suggests the replacement of copper interconnect with the multiwalled and bundles of single-walled carbon nanotubes for the sub-nanometer CMOS technologies.
\end{abstract}

Keywords: carbon nanotubes (CNTs), interconnects, single-walled CNT bundle, multiwalled CNT, interconnect modeling, $\mathrm{Cu}$ interconnects, CNT-FETs, integrated circuits.

\section{INTRODUCTION}

Nanometer CMOS technology especially in $22 \mathrm{~nm}$ and below is plagued due to performance degradation of conventional $\mathrm{Cu} / \mathrm{low}-\mathrm{k}$ dielectric as an interconnect material for gigascale integration. In one of the recent published research on interconnect technologies, Koo et al. [1] have mentioned the effect of scaling on surface and grain boundary scattering and electromigration in $\mathrm{Cu}$ interconnect [2] and in great detail degradation in its parameters such as the latency and power dissipation. Thus the need for other materials possibly substituting $\mathrm{Cu} /$ low-k dielectric interconnections has brought forward other novel interconnect technologies for next generation VLSI interconnects. Optical interconnects have already been suggested for on-chip integration [3-5] but still face serious integration problems. Among newer and novel VLSI interconnection technologies, carbon nanotubes (CNTs) and graphene nanoribbons (GNR) have emerged promising candidates for next generation VLSI interconnects [6-12]. An excellent review of these technologies has been presented in one of the recent publications of Li et al. [13]. Though optical interconnect is still being investigated due to its inherent advantages over the $\mathrm{Cu}$ interconnect, other new technologies such as the capacitively driven low-swing interconnect (CDLSI) have been also evolved [1]. In search for novel technologies, no such material has aroused so much interest other than carbon nanomaterials since the discovery of carbon nanotube in 1991 by Iijima [14].

The one-dimensional carbon nanotube has excellent electrical, mechanical and thermal properties $[15,16]$ which has made the CNT one of the promising materials for applications 
in nanoelectronics $[6,7,10,17]$ and micro/nano-systems [18]. In nanoelectronics, CNT-FET is very promising in design of emerging logic devices for nano scale integration and there is a noticeable amount of published and on going research on understanding current transport in CNT-FETs and developing models for use in circuit simulators [19-28]. In micro/nanosystems, both the CNT and CNT-FET are very promising as sensors for detecting chemicals, gases at molecular levels $[18,29-33]$. Carbon nanotube carries a current density of $\sim 10^{10}$ $\mathrm{A} / \mathrm{cm}^{2}$ which is higher by a two to three orders of magnitude in $\mathrm{Cu}$. Its mean free path is in micrometer range compared to $\sim 40 \mathrm{~nm}$ mean free path in $\mathrm{Cu}$. The large mean fee path in CNT allows a ballistic transport over a wider range of micrometers resulting in reduced resistivity, and strong atomic bonds [34] provide tolerance to electromigration [1, 9]. Higher thermal conductivity makes the CNT suitable for use in tall vias of 3D ICs $[13,35,36]$. Electrical performance of single and bundled carbon nanotubes have been studied in the work of Plombon et al. [37], Yao et al. [38] and Nougaret et al. [39]. Recently Sarto and Tamburrano [40] have presented analytical derivation of multiwalled carbon nanotubes from the multiconductor transmission line model. Properties of carbon nanomaterials relevant to VLSI interconnects which include single-walled carbon nanotube (SWCNT), multiwalled carbon nanotube (MWCNT), GNR and comparison with the properties of $\mathrm{Cu}$ interconnect are summarized in the work of Li et al. [13]. GNR is a recent addition to the interconnect technology since the discovery of 2D graphene in 2004 [41, 42] and the methods of fabricating GNR are still being developed.

The present work focuses on the carbon nanotube and its electrical modeling as interconnects in VLSI. Single-walled, multiwalled and bundle of CNTs as interconnects have been considered. Basic CNT-FET circuits have been analyzed with three types of CNT interconnections and simulated in Verilog-AMS. In Sec. 2, equivalent circuit models of CNT interconnections have been discussed. In Sec. 3, performance of CNT interconnects have been evaluated and compared with $\mathrm{Cu}$ interconnects. Section 4 presents conclusion.

\section{CARBON NANOTUBE INTERCONNECT MODELING}

Since the discovery of carbon nanotubes and its outstanding electrical, mechanical and thermal properties this material has been sought after $\mathrm{Cu}$ as interconnects for VLSI [43, 44]. A good amount of research has been conducted in understanding and modeling of current transport in SWCNT, SWCNT bundles and MWCNTs [45-54]. Fetter [55, 56] and Maffucci et al. [57] have investigated electron transport along the CNT and proposed a twodimensional fluid model. In these models [55-57], electron-electron correlation, which is significant in CNTs [58-60], has not been considered. Burke [61, 62] regards that electrons are strongly correlated when they transport along the CNT and have proposed a transmission line model based on the Lüttinger liquid theory [63], which describes interacting electrons (or other fermions) in one-dimensional conductor.

In a recent work [64], we have made modification in two-dimensional fluid model to include electron-electron repulsive interaction and built a semi-classical one-dimensional fluid model. In this model, metallic single-walled carbon nanotube is considered and represented by a transmission line model. The SWCNT is regarded as a graphene sheet rolled to form a tube of infinitesimally thin layer. The conduction electrons are distributed on the lateral surface of the SWCNT cylindrical shell. The electrons are embedded in a rigid uniform positive charge background with a uniform surface carrier concentration. Besides its accuracy compared with two-dimensional fluid model and Lüttinger liquid theory, one-dimensional fluid model is simple in mathematical modeling and easier to extend for electronic transport modeling of multiwalled carbon nanotubes and single-walled carbon nanotube bundles as interconnections. In the following sub-sections, we will describe theoretical modeling of SWCNT, MWCNT and SWCNT bundles for interconnections. 


\subsection{One dimensional fluid model}

A SWCNT is an atom thick sheet of graphite (called graphene) rolled up into a seamless cylinder with diameter of the order of a nanometer. This results in a nanostructure where the length-to-diameter ratio exceeds 10,000. Since carbon nanotubes are constructed of hexagonal networks, the carbon atoms contain $s p^{2}$ hybridization. There are four valence electrons for each carbon atom. The first three electrons belong to the $\sigma$ orbital and are at energies $2.5 \mathrm{eV}$ below the Fermi level; therefore, these electrons do not contribute to the conduction. The fourth valence electron, however, is located in the $\pi$ orbital, which is slightly below the Fermi level; therefore, this electron is very likely to control conduction and transport properties. This electron corresponds to the valence band of the energy band diagram. The anti-bonding $\pi$ orbital is slightly above the Fermi level, which corresponds to the conduction band in an energy band diagram. Depending upon the direction in which the graphene sheet is wrapped; the CNT can be metallic or semiconducting.

If we regard the graphene sheet which is rolled to form a CNT is infinitesimally thin, then the conduction electrons are distributed on the lateral surface of the CNT cylinder shell and the electrons are embedded in a rigid uniform positive charge background with a uniform surface number density. Thus, the motion of electrons is confined to the surface. Furthermore, electrical charge neutrality requires that in equilibrium the conduction electron charge density precisely cancels with that of the background positive ions. According to this analysis, fluid model could be utilized to study the electron transport along the CNT. This model is shown in Fig. 1. The cylinder shell radius is $r$ and length is $l$. The cylinder axis is oriented along the $z$ axis of the reference system. Two assumptions have been made to utilize this model. One is the electrons can only move along the $z$-axis; other is that all other fluid variables, such as the tangential component of the electric field to the lateral surface, $s^{\prime}$ of the nanotube, are almost uniform in the cross section plane of the CNT. These two assumptions are valid if both the nanotube length and the smallest wavelength of the electromagnetic field are much greater than the nanotube radius $[65,66]$.

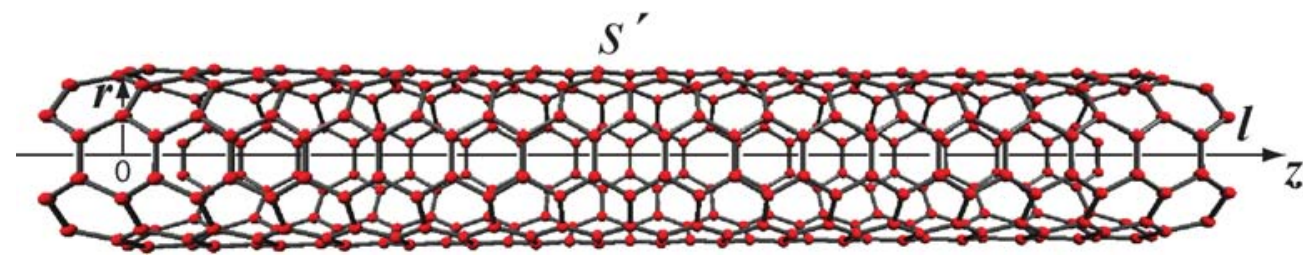

Fig. 1. Geometry of a single-walled carbon nanotube (SWCNT).

If we neglect heat transfer and viscosity in the CNT, Euler's equation with Lorentz Force term [55-57] can be used to describe transport of electrons in a CNT as follows,

$$
m N\left(\frac{\partial}{\partial t}+\vec{V} \cdot \nabla\right) \vec{V}=-\nabla \vec{P}-e N \vec{E}-m N v \vec{V},
$$

where $N(\vec{R}, t)$ is the electron three-dimension carrier density, $\vec{V}(\vec{R}, t)$ is the electron mean velocity, $\vec{R}$ is the position vector, $\vec{P}$ is the pressure, $m$ is the electron mass, $e$ is the electronic charge and $\vec{E}$ is electric field. The last term on the right hand side represents the effect of scattering of electrons with the positive charge background and $v$ is the electron relaxation frequency.

It should be noted that Lorentz force term, which belongs to body force terms in fluid dynamics, is a source momentum [67]. The external electric field provides both the potential and kinetic energy to the fluid. As a result, one-dimensional fluid model can be expressed as follows [64], 


$$
m n\left(\frac{\partial}{\partial t}+v_{z} \frac{\partial}{\partial z}\right) v_{z}=-\frac{\partial p}{\partial z}-e n\left\{\left.(1-\alpha) E_{z}\right|_{s^{\prime}}\right\}-m n v_{z},
$$

where $n$ is the electron density in this one-dimensional system, $v_{z}$ is the electron mean velocity in $z$ direction, $p$ is the pressure in one-dimensional system. $\boldsymbol{\varepsilon}_{z}$ is electric field in $z$ direction. In one-dimensional fluid model, $\alpha$ is defined as [64],

$$
\alpha \equiv \frac{E_{z P}}{E_{z}}=\frac{E_{P}}{E}=\frac{E_{P}}{E_{K}+E_{P}},
$$

where $\boldsymbol{\varepsilon}_{z P}$ is the part of the electrical field which provides potential energy to electrons in $z-$ direction. $E$ is the total energy of electrons. $E_{P}$ and $E_{K}$ are the potential and kinetic energies of electrons, respectively.

The fluid model described by Eq. (2) assumes flow of one-dimensional electron fluid under the low external electric fields, $\boldsymbol{\varepsilon}_{2}$. Yao, et al. [38] and Park et al. [68] have studied electronic transport in SWCNTs using low and high resistance contacts under small and large bias-voltages and attempted to explain the conductance behavior from electron-phonon scattering. Yao et al. [38] have also observed linear I-V characteristics from measurement on samples using low resistance contacts with slight deviation near $5 \mathrm{~V}$ from the linear behavior in some samples. Since current VLSI circuits use low voltage nanometer CMOS technologies where bias voltage is below $1.5 \mathrm{~V}$, deviation in I-V relationship of CNT interconnect toward saturation at large bias-voltages should not be the cause of concern and so the applicability of Eq. (2) for CNT interconnects using low resistance contacts.

The difference between our one-dimensional fluid model described in Eq. (2) with twodimensional fluid model [55-57] is the Lorentz force term. In a two-dimensional electron fluid [55], total energy is equal to the kinetic energy. In a graphene sheet, the external electrical field drifts electrons in $z$ direction while electrons can also distribute in perpendicular $(y)$ direction to form a two-dimensional fluid. This means that the whole energy provided by the external electrical field equals the two-dimensional electron fluid kinetic energy. However, when the graphene sheet is rolled to form a carbon nanotube, which is a quasi onedimensional system, $y$ direction shrinks into one point and the distributed electrons in $y$ direction in a two-dimensional system will be at the same point in one-dimensional system. As a result, the potential energy due to repulsive force among the electrons will be significant. The external electric field provides both the potential and kinetic energies to the onedimensional fluid.

\subsection{SWCNT interconnect modeling}

Equation (2) describes our one-dimensional fluid model which can be simplified in the following form [64],

$$
m n_{0} \frac{\partial v_{z}}{\partial t}=-\frac{\partial \delta p}{\partial z}-e n_{0}\left\{(1-\alpha) E_{z}\right\}-m n\{\operatorname{sgn}(l)\} \mathcal{v}_{z},
$$

where $l$ is the length of CNT and $\operatorname{sgn}(l)$ is the sign function defined as follows:

$\operatorname{sgn}(l)=\left\{\begin{array}{lll}0 & \text { if } & l<l_{m f p} \\ 1 & \text { if } & l \geq l_{m f p}\end{array}\right.$, since the mean free path, $l_{m f p}$ of electrons in a CNT is in micron range, the last term in Eq. (4) approaches to zero if $1<l_{m f p}$.

There are two channels in a CNT and two different spin electrons in each channel. We can consider that there are four electrons at the same point in one-dimensional CNT. We can then 
calculate the potential energy by moving these four electrons from $\infty$ to the same point of the CNT within $\sim 1 \mathrm{~nm}$ diameter. The potential energy can be defined as follows [69]:

$$
E_{P}=\sum_{n=2}^{4}(n-1) \times \frac{e^{2}}{2 \pi \varepsilon_{0}} \frac{1}{d} \approx 18 e V,
$$

where $d$ is the diameter of the CNT.

We assume that the velocity of these four electrons equals to the Fermi velocity. As a result, the kinetic energy is given by,

$$
E_{K}=4 \times \frac{1}{2} m v_{F}^{2} \approx 7 \mathrm{eV}
$$

where $v_{F}=3 \gamma_{0} b / 2 \hbar=8 \times 10^{5} \mathrm{~m} / \mathrm{s}$ is the Fermi velocity. $\gamma_{0}$ is the characteristic energy of the graphene lattice and is equal to $2.7 \mathrm{eV}$ and $\hbar$ is Planck constant. The inter-atomic distance, $b$ in a CNT is $0.142 \mathrm{~nm}$ [57]. Substituting Eqs. (5) and (6) into Eq. (3), we obtain $\alpha \approx 0.7$.

The equation relating current density, charge density and electric field can be described as follows [64],

$$
\frac{\partial j(z, t)}{\partial t}+v j+u_{e}^{2} \frac{\partial \sigma(z, t)}{\partial z}=\frac{e^{2} n_{0}}{m}(1+\alpha) E_{z} .
$$

Equation (7) is the current transport equation for a one-dimension electron fluid in a metallic CNT. The third term on the left-hand side of Eq. (7) can be neglected since metallic CNT is a good conductor.

We consider a metallic SWCNT above a perfect conducting plane and assume that the propagating EM wave is in quasi-TEM mode. Following the analysis in the work of Maffucci et al. [57], we obtain,

$$
\mathrm{E}_{z}=R i+L_{K} \frac{\partial i}{\partial t}+\frac{1}{C_{Q}} \frac{\partial q}{\partial z}
$$

where $R \equiv L_{K} \operatorname{sgn}(l) v$ is the resistance of CNT per unit length. $L_{K} \equiv \pi \hbar / 4 e^{2} v_{F}$ is the kinetic inductance per unit length and $C_{Q} \equiv 1 / L_{K} u_{e}^{2}$ is the quantum capacitance per unit length. $u_{e}=v_{F} / \sqrt{1-\alpha}$, is the thermodynamic speed of sound of the electron fluid under neutral environment.

The magnetic inductance and electrostatic capacitance per unit length of a perfect conductor on a ground plane is given by $[57,70]$,

$$
\begin{gathered}
L_{M}=\frac{\mu}{2 \pi} \cosh ^{-1}\left(\frac{h}{r}\right) \approx \frac{\mu}{2 \pi} \ln \left(\frac{h}{2 r}\right), \\
C_{E}=\frac{2 \pi \varepsilon}{\cosh ^{-1}(h / r)} \approx \frac{2 \pi \varepsilon}{\ln (h / r)},
\end{gathered}
$$

where $h$ is the distance of CNT to the ground plane and $r$ is the radius of a SWCNT. Equations (9) and (10) are accurate enough for $h>4 r$. The magnetic inductance is ignorable compared with kinetic inductance [64]. 


\subsection{MWCNT interconnection modeling}

MWCNTs have diameters in a wide range of a few to hundreds of nanometers. It has been shown that all shells of MWCNT can conduct if they are properly connected to contacts [7173] and the contact resistance could reach tens of ohms, a much lower value than that of SWCNT. Naeemi et al. [52] have shown that MWCNTs can have conductivities several times larger than that of $\mathrm{Cu}$ or SWCNT bundles for long length interconnects.

The number of shells in MWCNTs varies. The spacing between shells in a MWCNT corresponds to van der Waals distance between graphene layers in graphite, $\delta \approx 0.34 \mathrm{~nm}$ [74]. The number of metallic shells in a MWCNT can be calculated as follows:

$$
M=\beta\left[1+\frac{D_{1}-D_{N}}{2 \delta}\right],
$$

where $D_{l}$ and $D_{N}$ are the outermost and innermost shell diameters, respectively. The square bracket term is a floor function and the factor $\beta$ is the ratio of metallic shells to total shells in a MWCNT. Statistically, one-third of the shells are going to be metallic and the rest semiconductor for $D_{l} \leq 10 \mathrm{~nm}[74,75]$. For $D_{l}>10 \mathrm{~nm}, \beta$ increases due the interaction between adjacent shells for the MWCNT [74].

In one-dimensional fluid model [64], we regard the graphene sheet which is rolled to form a CNT is to be infinitesimally thin. The conduction electrons are then distributed over the lateral surface of the CNT cylinder shell and electrons are embedded in a rigid uniform positive charge background with a uniform surface number density. Thus, the motion of electrons is confined to the surface. Furthermore, electrical charge neutrality requires that in equilibrium the conduction electron charge density precisely cancels with that of the background positive ions. Since the van der Waals force between the carbon atoms in different shells in MWCNTs is negligible compared to valance band between the carbon atoms in the same shell [76], the one-dimensional fluid model described by Eq. (2) can be applied to each shell of the MWCNT with modification because the electron-electron interaction in MWCNT is different from that in SWCNT, which means the parameter $\alpha$ needs to be recalculated.

In addition, two assumptions are made: the electrons can only move along the $z$-axis; all other fluid variables, such as the tangential component of the electric field to the nanotube surface are almost uniform in the cross section plane of the shells in MWCNT. These two assumptions are valid if both the nanotube length and the smallest wavelength of the electromagnetic field are much greater than the nanotube radius $[65,66]$.

We assume that the velocity of these electrons equals the Fermi velocity. As a result, the kinetic energy is given by,

$$
E_{K}=4 M \times \frac{1}{2} m v_{F}^{2} \approx 7 M \mathrm{eV} .
$$

There are two channels in each shell of MWCNT and two different spin electrons in each channel. So we consider that there are four electrons at the same point in each shell of MWCNT. We can then calculate the potential energy by moving these $4 M$ electrons from $\infty$ to the same point of the MWCNT. We first consider moving every four electrons into one shell of the MWCNT. The potential energy can be obtained as follows [64, 70]:

$$
E_{P}=\sum_{j=1}^{M}\left[\sum_{i=2}^{4}(i-1) \frac{e^{2}}{2 \pi \varepsilon_{0}} \frac{1}{d_{j}}\right]=6 M \frac{e^{2}}{2 \pi \varepsilon_{0}} \sum_{j=1}^{M} \frac{1}{d_{j}},
$$

where $d_{j}$ is diameter of shell number $j$. 
We then consider moving all shells from $\infty$ to adjacent shells to construct a MWCNT. The potential energy can be calculated using following equation:

$$
E_{P}=6 M \frac{e^{2}}{2 \pi \varepsilon_{0}} \sum_{j=1}^{M} \frac{1}{d_{j}}+\sum_{j=2}^{M} 4(j-1) \times \frac{4 e^{2}}{2 \pi \varepsilon_{0}} \frac{1}{d_{j}}=6 M \frac{e^{2}}{2 \pi \varepsilon_{0}} \sum_{j=1}^{M} \frac{1}{d_{j}}+\frac{16 e^{2}}{2 \pi \varepsilon_{0}} \sum_{j=2}^{M} \frac{j-1}{d_{j}},
$$

The parameter $\alpha$ for the MWCNT can be calculated using Eq. (3). For example, if $D_{I}=10 \mathrm{~nm}$, $D_{N}=1 \mathrm{~nm}$ and $\beta=1$ then $\alpha \approx 0.99$.

Following the derivation in Ref. 64, we can obtain an equation for each shell in a MWCNT,

$$
\mathrm{E}=R i+L_{K} \frac{\partial i}{\partial t}+\frac{1}{C_{Q}} \frac{\partial q}{\partial z}
$$

where $R \equiv L_{K} \operatorname{sgn}(l) v$ is the resistance of each shell in a MWCNT per unit length. $L_{K} \equiv \pi \hbar / 4 e^{2} v_{F}$ is the kinetic inductance per unit length of each shell. $C_{Q} \equiv 1 / L_{K} u_{e}^{2}$ is the quantum capacitance per unit length of each shell. $u_{e}=v_{F} / \sqrt{1-\alpha}$, is the thermodynamic speed of sound of the electron fluid under a neutral environment.

The magnetic inductance per unit length of each shell can also be calculated using Eq. (9). In a SWCNT, magnetic inductance is neglected compared with kinetic inductance; therefore, it can also be neglected in each shell of a MWCNT.

The outermost shell shields inner shells from the ground plane; therefore, the electrostatic capacitance $C_{E}$ does not exist in inner shells. However, there exists electrostatic capacitance, $C_{S}$ between the neighboring metallic shells and its value is given by [70,77]:

$$
C_{S}=\frac{2 \pi \varepsilon_{0}}{\ln \left(D_{i} / D_{j}\right)}
$$

where $\varepsilon_{0}$ is the permittivity of vacuum, $D_{i}$ and $D_{j}$ are the diameters of the $i^{\text {th }}$ and $j^{\text {th }}$ metallic shells, respectively and $i<j$.

We assume that the outermost shell is metallic. In a recent work [77], we have derived an equivalent circuit of a metallic MWCNT interconnect as shown in Fig. 2. It is simplified as shown in Fig. 3 by considering that the RLC parts of all inner shells are identical. If we assume that there are no variation in distributed parameters, $R$ and $L_{K}$ then $\mathrm{R}$ and $L_{K}$ are same for each shell. The potential across components of each shell in a MWCNT is equal. As a result, a simplified equivalent circuit of a MWCNT interconnect can be derived as shown as Fig. 4 . $R_{C}$ in Figs. $2-4$ is the contact resistance and its ideal quantum value is $3.2 \mathrm{k} \Omega$ per shell [62].

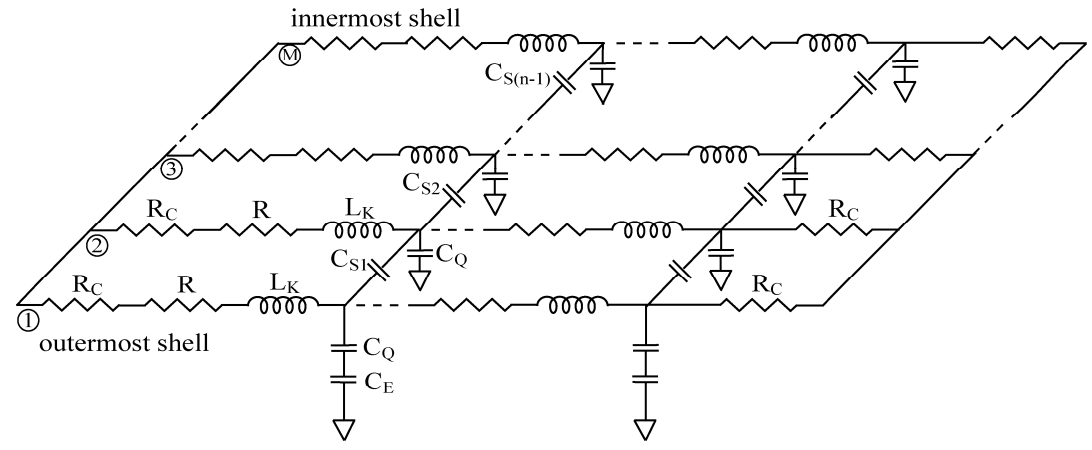

Fig. 2. Equivalent circuit of a metallic MWCNT interconnect. 


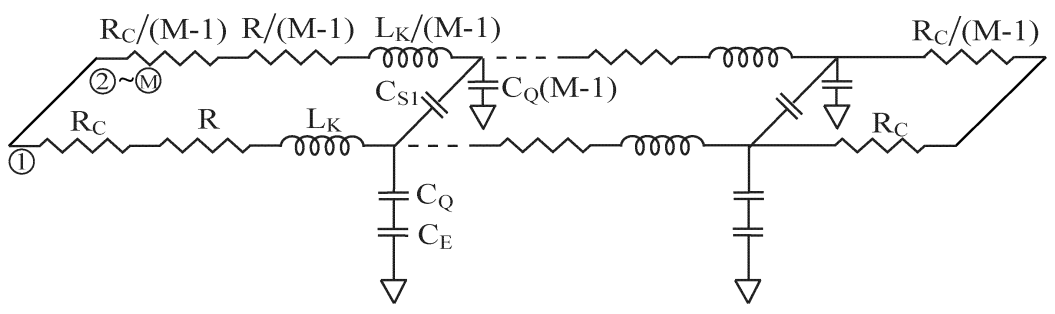

Fig. 3. Simplified equivalent circuit of a metallic MWCNT interconnect.

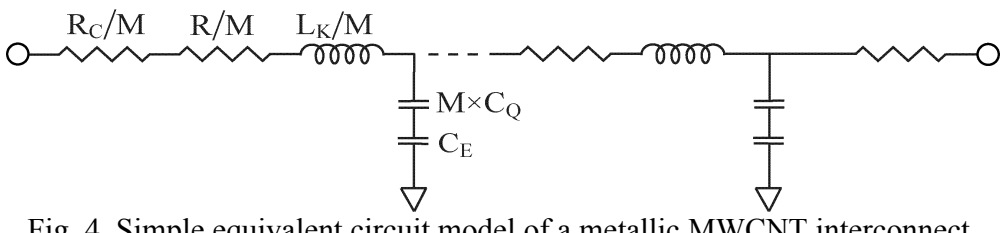

The values of $C_{E}$ and $C_{Q}$ are on same order. $C_{Q}$ of all metallic shells is in parallel and then serial with $C_{E}$. As a result, $C_{Q}$ can be neglected if $M$ is large. Therefore, capacitance of MWCNT interconnect is smaller than that of SWCNT. In addition, the resistance and inductance of all metallic shells are parallel and M times smaller than that of SWCNT.

\subsection{SWCNT bundle interconnection modeling}

Carbon nanotubes can also be fabricated as a bundle which means CNTs in a bundle are parallel to each other. The spacing between carbon nanotubes in the bundle is due to the van der Waals forces between the atoms of adjacent nanotubes [78]. One of the most critical challenges in realizing high-performance SWCNT-based interconnects is controlling the proportion of metallic nanotubes in the bundle. Current SWCNT fabrication techniques cannot effectively control the chirality of the nanotubes in the bundle [17, 79]. Therefore, SWCNT bundles have metallic nanotubes that are randomly distributed within the bundle. Avouris et al. [17] and Liebau et al. [79] have shown that metallic nanotubes are distributed with a probability, $\beta=1 / 3$ in a growth process. The proportion of metallic nanotubes can; however be potentially increased using techniques introduced in Refs. 80 and 81 .

Figure 5 shows the cross-section of a SWCNT bundle. In Fig. 5, $d$ is the diameter of SWCNT, $\delta=0.34 \mathrm{~nm}$ is the spacing between the SWCNT in the bundle and corresponds to the van der Waals distance between graphene layers in graphite. $d_{b}=\delta+d$ is the distance between adjacent SWCNT. Since van der Waals forces between carbon atoms in adjacent SWCNTs are negligible compared to valance band between carbon atoms in the SWCNT [76], influence of adjacent SWCNTs on transport of electrons in SWCNT can be considered to be very small. Therefore, the one-dimensional fluid model described by Eq. (2) can be applied to the each SWCNT in the bundle with some modification. The electron-electron interaction in SWCNT bundle is different from that in a SWCNT, the parameter $\alpha$ needs to be recalculated to account for this effect.

Consider one of the SWCNTs in the bundle, we assume that the electrons in this SWCNT will only be affected by the electrons in the adjacent metallic SWCNTs and semiconducting SWCNTs have no effect on the conductance of the bundle. To calculate the potential energy, we first consider the potential energy of each SWCNT and then consider moving SWCNT adjacent to each other to construct a SWCNT bundle. Average potential energy of electrons in a SWCNT bundle can then be described by the following equation: 


$$
E_{P}=6 \frac{e^{2}}{2 \pi \varepsilon_{0}} \frac{1}{d}+\sum_{i=1}^{\Gamma} \frac{16 e^{2}}{2 \pi \varepsilon_{0}} \frac{1}{d_{b}}
$$

where $\Gamma$ is the average number of metallic SWCNTs neighboring to a single SWCNT. As shown in Fig. 5, e.g., the number of SWCNTs neighboring to the corner SWCNT is 2, the number of SWCNTs neighboring to the edge SWCNT is 4 and the number of SWCNTs neighboring to the inside SWCNT is 6 . Therefore, $\Gamma=\beta\left[\frac{6 N_{x} N_{y}-4 N_{x}-4 N_{y}-2\left[N_{y} / 2\right]}{N_{x} N_{y}-2\left[N_{y} / 2\right]}\right]$, where square brackets is the floor function.

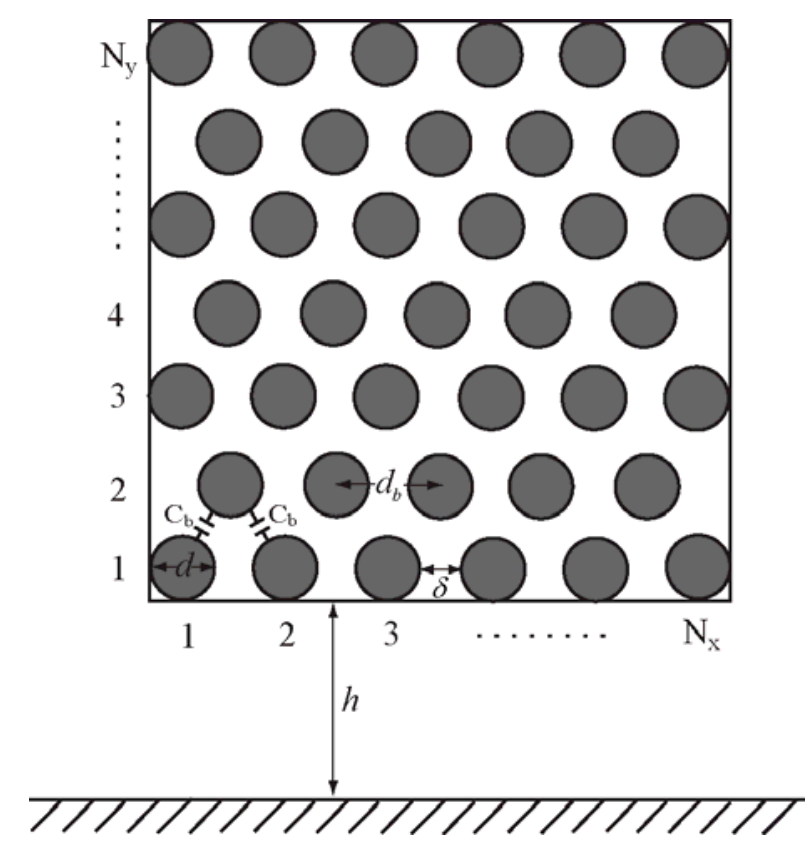

Fig. 5. Cross-section of a SWCNT bundle interconnect.

The kinetic energy of the electrons in a SWCNT is calculated as follows [64]:

$$
E_{K}=4 \times \frac{1}{2} m_{e} v_{F}^{2} \approx 7 \mathrm{eV} .
$$

Now the parameter $\alpha$ for SWCNT bundle can be calculated using Eq. (3). Total number of metallic SWCNTs is $N=\beta\left(N_{x} N_{y}-\left[N_{y} / 2\right]\right)$. Following the derivation in Ref. 64, we obtain an equation for each single SWCNT in a bundle,

$$
\mathrm{E}=R i+L_{K} \frac{\partial i}{\partial t}+\frac{1}{C_{Q}} \frac{\partial q}{\partial z}
$$

where $R \equiv L_{K} \operatorname{sgn}(l) v$ is the resistance per unit length of a SWCNT in a SWCNT bundle.

In Eq. (19), $L_{K} \equiv \pi \hbar / 4 e^{2} v_{F}$ is the kinetic inductance per unit length of a SWCNT in a bundle and $C_{Q} \equiv 1 / L_{K} u_{e}^{2}$ is the quantum capacitance per unit length of a SWCNT in a 
bundle. $u_{e}=v_{F} / \sqrt{1-\alpha}$ is the thermodynamic speed of sound of the electron fluid under a neutral environment.

The magnetic inductance per unit length of each SWCNT can also be calculated using Eq. (9). In a single SWCNT, magnetic inductance is neglected compared with the kinetic inductance. It can also be neglected in each SWCNT of a bundle since magnetic inductance is comparable to the kinetic conductance when the number of SWCNTs is above 4000 in a bundle [48], while this number is only about 500 for the bundle with $22 \mathrm{~nm} \times 44 \mathrm{~nm}$ size. This size of the bundle corresponds to interconnections in $22 \mathrm{~nm}$ technology.

The SWCNTs at the bottom level shield upper levels SWCNT from the ground plane. Therefore, the electrostatic capacitance, $C_{E}$ does not exist in the upper SWCNTs. However, there exists electrostatic capacitance per unit length, $C_{b}$, between the neighboring metallic SWCNTs and its value can be calculated as follows [70],

$$
C_{b}=\frac{\pi \varepsilon_{0}}{\ln \left(\frac{d_{b}}{d}+\sqrt{\left(\frac{d_{b}}{d}\right)^{2}-1}\right)} .
$$

Figure 6 shows the equivalent circuit of a SWCNT bundle interconnect [82] where $N_{a}$ is the number of upper level SWCNTs and $N_{b}$ is the number of bottom level SWCNTs. For a SWCNT bundle, we assume that all SWCNTs in the bundle are identical and each SWCNT has the same potential across it $[83,84]$. The circuit can be further simplified as shown in Fig. 7. The capacitance, $C_{b}$ has no effect on the circuit behavior and $\beta N_{x} \times C_{E}$ can be regarded as an electrostatic capacitance between SWCNT bundle and the ground plan.

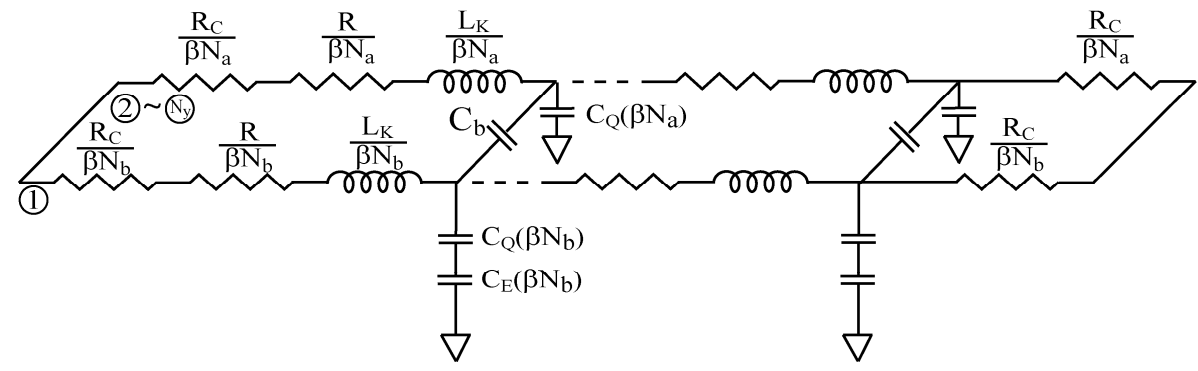

Fig. 6. Equivalent circuit of a SWCNT bundle interconnect.

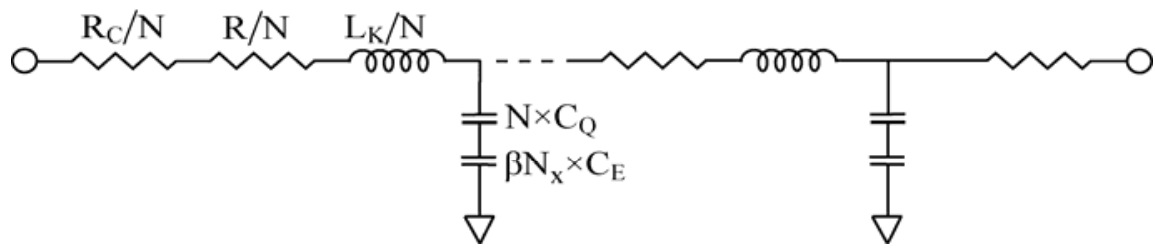

Fig. 7. Simplified equivalent circuit of a SWCNT bundle interconnect.

The values of $C_{E}$ and $C_{Q}$ are nearly the same in magnitude. $C_{Q}$ of all metallic SWCNTs are in parallel and then is serial with $C_{E}$, as a result, $C_{Q}$ can be neglected if $N$ is large. Therefore, capacitance of the SWCNT bundle interconnects is smaller than that of a SWCNT. In addition, the resistance and inductance of all metallic SWCNTs are in parallel in the bundle and $N$ times smaller than that of a SWCNT. 


\section{PERFORMANCE OF CARBON NANOTUBE INTERCONNECTS}

In Sec. 2.3, we have extended one-dimensional fluid model for the modeling of MWCNT interconnects. To validate the model of MWCNT interconnect, we have compared the calculated resistance of MWCNT interconnect with the corresponding measured resistance from the work of Nihei et al. [71] and Li et al. [72]. The results of the comparison of calculated and measured resistances are summarized in Table 1.

Table 1: A comparison of calculated and measured resistances of MWCNT interconnects

\begin{tabular}{|c|c|c|c|c|c|c|}
\hline \multirow[b]{2}{*}{ References } & \multicolumn{4}{|c|}{ MWCNT Physical Parameters } & \multicolumn{2}{|c|}{ 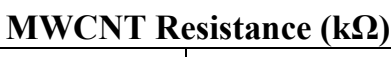 } \\
\hline & $\begin{array}{c}\text { Length } \\
(\mu \mathrm{m})\end{array}$ & $\begin{array}{c}\mathbf{D}_{1} \\
(\mathbf{n m})\end{array}$ & $\begin{array}{c}\mathbf{D}_{\mathbf{n}} \\
(\mathbf{n m})\end{array}$ & $\begin{array}{c}I_{m f p} \\
(\mu \mathrm{m})\end{array}$ & Me & Model \\
\hline Nihei et al. [71] & 2 & 10 & 3.88 & $<1$ & 1.60 & 1.90 \\
\hline Li et al. [72] & 25 & 100 & 50 & $>25$ & 0.035 & 0.042 \\
\hline
\end{tabular}

CNT interconnects have great potential in extending the operation of circuits to higher speeds and frequencies. For applications requiring high frequencies where newer interconnect technologies and materials for interconnect are being explored, it is important to study twoport scattering parameters. Slepyan et al. [85] have conducted studies on scattering of electromagnetic waves by a semi-infinite CNT in optical regime. In this work, we focus on studying 2-port network scattering (S) parameters by CNT for interconnect applications on a chip.

Figure 8 shows the schematic of a 2-port network used in study of S-parameters. In Fig. 8, interconnect can be $\mathrm{Cu}$ or $\mathrm{CNT}$ and $R_{S}$ is the terminal impedance. We have utilized the process parameters from the 2016 node $(22 \mathrm{~nm}$ technology), assumed a $22 \mathrm{~nm}$ diameter of MWCNT of $22 \mathrm{~nm}$ width and $44 \mathrm{~nm}$ thickness of SWCNT bundle [86]. If we assume diameter of the innermost shell in a MWCNT is to be $1 \mathrm{~nm}$ then there are nearly 30 shells in $22 \mathrm{~nm}$ MWCNT. If we assume diameter of a SWCNT in a bundle is to be $1 \mathrm{~nm}$ then there are nearly 500 SWCNTs in $22 \mathrm{~nm}$ (width) $\times 44 \mathrm{~nm}$ (thickness) bundle following Fig. 5. The resistivity and capacitance of $\mathrm{Cu}$ were taken from ITRS 2007 [86]. The inductance of $\mathrm{Cu}$ wire can be modeled by the following equation [87],

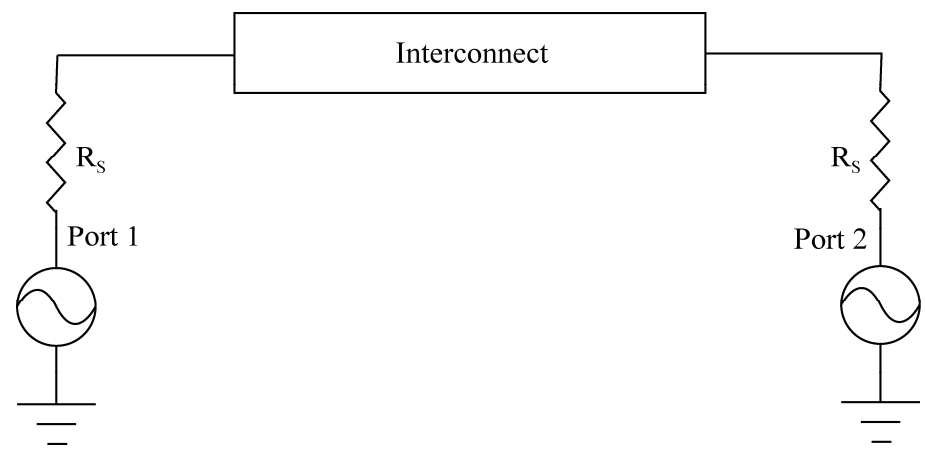

Fig. 8. Schematic of a 2-port network of interconnects.

$$
L=2 \times 10^{-4} l\left(\ln \frac{2 l}{w+t}+0.5+\frac{w+t}{3 l}\right),
$$

where $l, w$ and $t$ are the length, width and thickness of the $\mathrm{Cu}$ wire in micrometers. 
In a recent work [64], we have used 2-port network analysis to compare S-parameters of SWCNT and $\mathrm{Cu}$ interconnects in $22 \mathrm{~nm}$ technology [86] as shown in Figs. 9 and 10. In the analysis of SWCNT interconnect, the terminal impedance is assumed to be equal to its contact resistance; and in the analysis of $\mathrm{Cu}$ interconnect, the terminal impedance is assumed to be equal to its distributed resistance. Figures 9 and 10 show $S_{21}$ and $S_{11}$ parameters for $\mathrm{Cu}$ and SWCNT interconnects of $0.1,1,10$ and $100 \mu \mathrm{m}$ lengths. Since $\mathrm{Cu}$ has higher resistance and capacitance than that of SWCNT, single-walled CNT interconnect has larger $3 \mathrm{~dB}$ bandwidth, higher transmission efficiency and lower reflection losses.

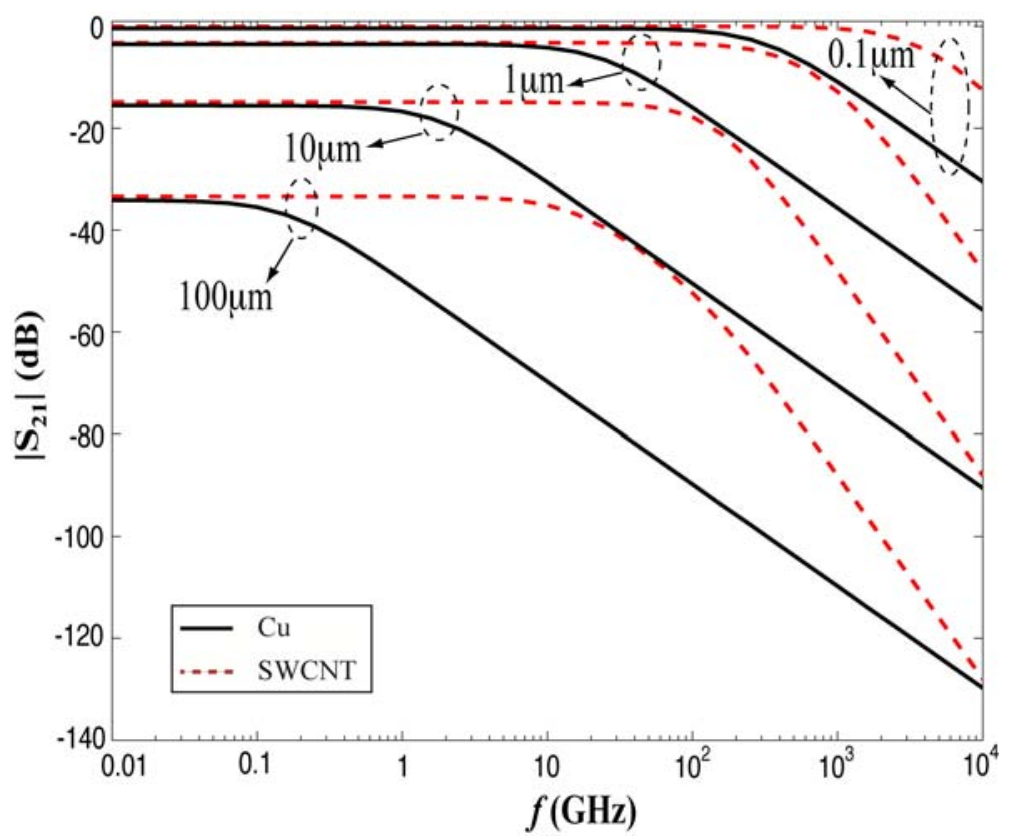

Fig. 9. $\mathrm{S}_{21}$ (amplitude) versus frequency of different lengths SWCNT and $\mathrm{Cu}$ interconnects.

SWCNT has very large contact resistance $[57,61,62]$ when used as interconnects, which limits its applications as interconnects for next generation integrated circuits. On the other hand MWCNT and CNT bundle give low contact resistance when used as the circuit interconnects [71-73, 88, 89]. Contact resistance in MWCNT and SWCNT bundle, however, will depend on the number of shells or SWCNTs being metallic. Close et al. [90-92] have demonstrated experimentally that MWCNT can function as an interconnect wire on a chip and successfully transmit $\mathrm{GHz}$ digital signals from one transistor to another. In Table 2, modeling parameters of MWCNT are compared with the equivalent model parameters from the quantum theory $[52,53]$. The difference is about $20 \%$. However, for large diameter, such as $100 \mathrm{~nm}$ diameter, the difference reaches to about $60 \%$. According to quantum theory, the number of channels increases significantly for large values of radius [84] and the semiconducting shells start contributing significantly to the number of conducting channels since their axial conductivity increases with increasing radius $[52,93]$. Our semi-classical one-dimensional fluid model assumes that the number of conducting channels in a single metallic CNT shell is fixed, 2, and 0 for semiconducting shells. Therefore, the difference in values of parameters between our model and quantum theory increases with the increase in diameter of MWCNTs. On the other hand, the parameter $\alpha$ decreases with the increase in diameter and quantum capacitance increases with the increase in diameter which is consistent with the quantum theory $[52,53]$. As a result, the difference in values of parameters 
calculated from our model and quantum theory is not very large for small diameter MWCNTs. The electrostatic capacitance is dependent on the geometry of the structure, it is, thus, considered same for one-dimensional fluid model and quantum model [53].

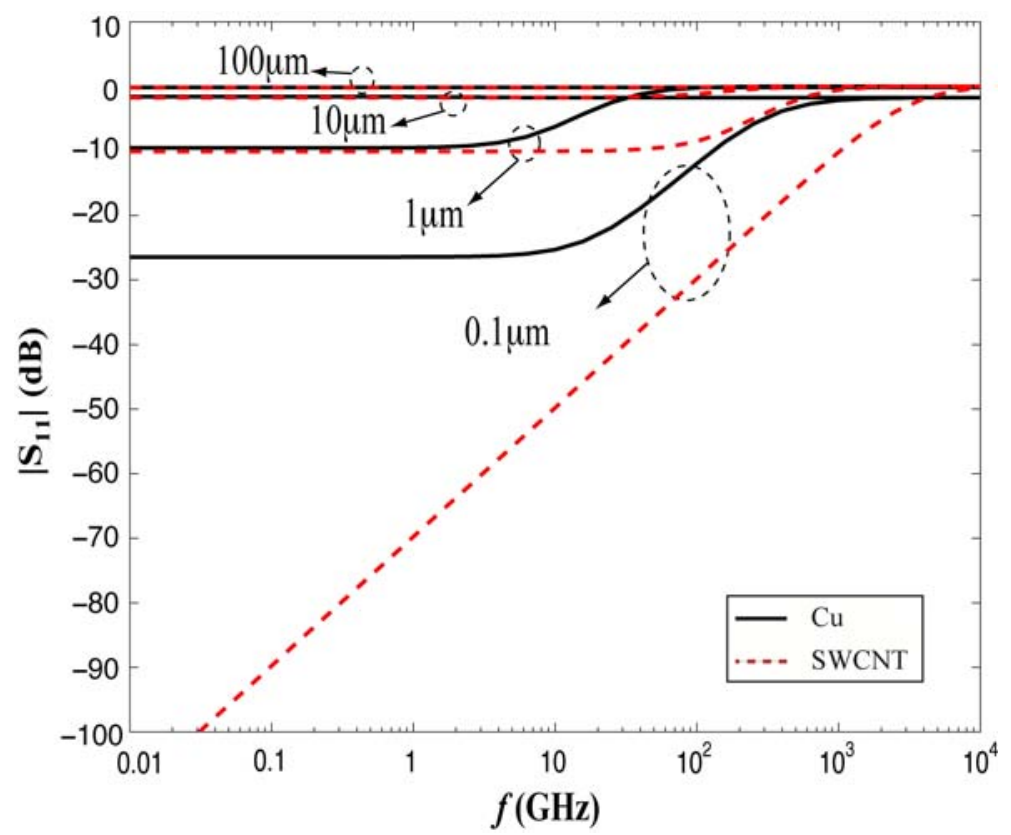

Fig. 10. $\mathrm{S}_{11}$ (amplitude) versus frequency for different lengths SWCNT and $\mathrm{Cu}$ interconnects.

Table 2: A comparison of MWCNT interconnect model parameters

\begin{tabular}{|c|c|c|c|c|c|c|c|c|}
\hline $\begin{array}{c}\text { MWCNT } \\
\text { Diameter } \\
(\mathbf{n m})\end{array}$ & $\begin{array}{c}\mathbf{R}_{\mathrm{C}} \\
\text { (cal) } \\
\mathrm{k} \Omega \\
\end{array}$ & $\begin{array}{l}\mathbf{R}_{\mathbf{C}} \\
{[\mathbf{5 3}]} \\
\mathbf{k} \boldsymbol{\Omega} \\
\end{array}$ & $\begin{array}{c}\mathrm{R} \\
(\mathrm{Cal}) \\
\mathrm{k} \Omega / \mu \mathrm{m} \\
\end{array}$ & $\begin{array}{c}\mathbf{R} \\
{[53]} \\
\mathrm{k} \Omega / \mu \mathrm{m} \\
\end{array}$ & $\begin{array}{c}\mathrm{L}_{\mathrm{K}} \\
\text { (cal) } \\
\mathbf{n H} / \mu \mathrm{m} \\
\end{array}$ & $\begin{array}{c}\mathrm{L}_{\mathrm{K}} \\
{[53]} \\
\mathrm{nH} / \mu \mathrm{m}\end{array}$ & $\begin{array}{c}\mathrm{C}_{\mathrm{Q}} \\
(\mathrm{cal}) \\
\mathrm{aF} / \mu \mathrm{m}\end{array}$ & $\begin{array}{c}\mathrm{C}_{\mathrm{Q}} \\
{[53]} \\
\mathrm{aF} / \mu \mathrm{m}\end{array}$ \\
\hline 18 & 0.81 & 1.05 & 0.81 & 1.05 & 1.00 & 1.31 & 1280 & 1160 \\
\hline 20 & 0.65 & 0.78 & 0.65 & 0.78 & 0.80 & 0.97 & 1600 & 1566 \\
\hline 22 & 0.65 & 0.72 & & & & 0.90 & 1600 & 1682 \\
\hline 25 & 0.54 & 0.55 & 0.54 & 0.55 & 0.67 & 0.68 & 1920 & 2228 \\
\hline 28 & 0.46 & 0.43 & 0.46 & 0.43 & 0.57 & 0.53 & 2240 & 2844 \\
\hline 32 & 0.40 & 0.34 & 0.40 & 0.34 & 0.50 & 0.42 & 2560 & 3622 \\
\hline 90 & 0.16 & 0.06 & 0.16 & 0.06 & 0.21 & 0.08 & 11080 & 19208 \\
\hline 100 & 0.11 & 0.04 & 0.11 & 0.04 & 0.17 & 0.05 & 17680 & 29845 \\
\hline
\end{tabular}

Figures 11 and 12 show $\mathrm{S}_{21}$ and $\mathrm{S}_{11}$ parameters and comparison with the corresponding Sparameters for MWCNTs calculated using model parameters from the work of Li et al. [53]. The dimensions used in comparison correspond to 18,22 and $32 \mathrm{~nm}$ diameters of the outermost shells of MWCNTs which also correspond to nanometer CMOS technologies. The length of MWCNTs used in calculations is $10 \mu \mathrm{m}$. Terminal impedance is set equal to contact resistance and $D_{1} / D_{N}=2$ and $\beta=1 / 3$. The parameters, $\mathrm{S}_{21}$ and $\mathrm{S}_{11}$ in both models differs by about $6 \%$ corresponding to $18 \mathrm{~nm}$ diameter and it is less than $6 \%$ for 22 and $32 \mathrm{~nm}$ diameters. 
The phase difference is negligible within the $3 \mathrm{~dB}$ bandwidths. It can, thus, be stated that the one-dimensional fluid model can be easily used in studying the performance of MWCNT interconnects.

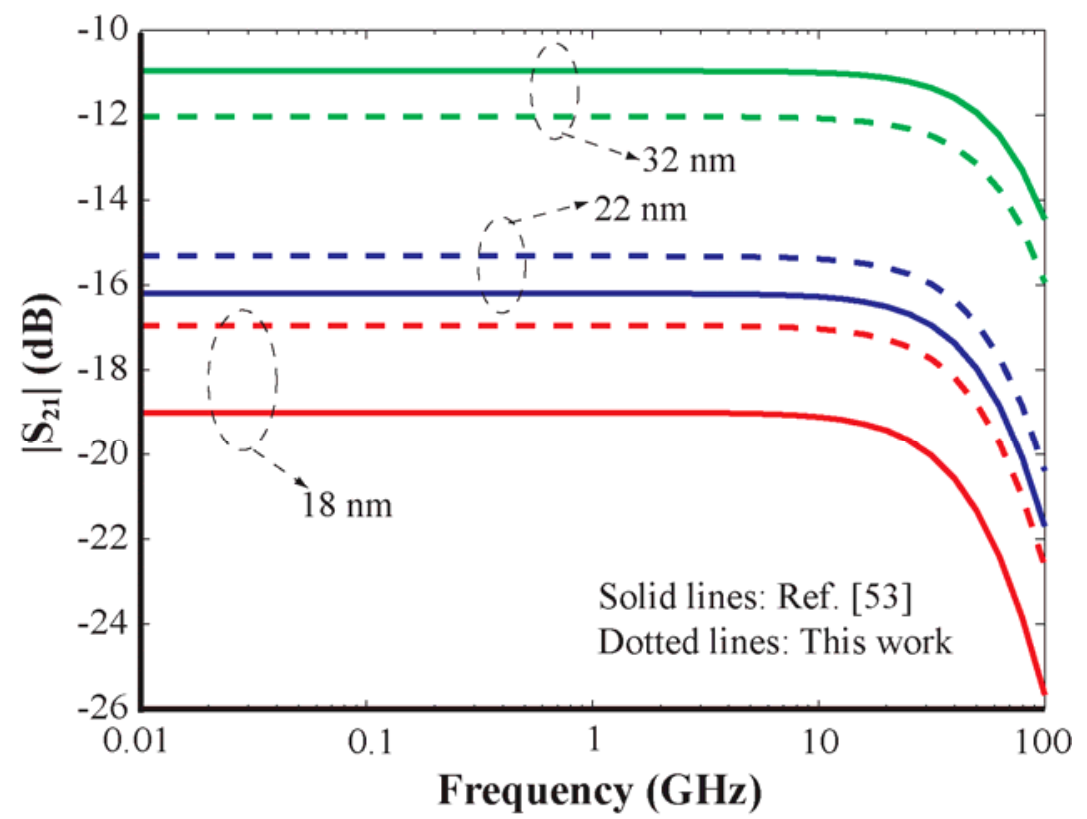

(a)

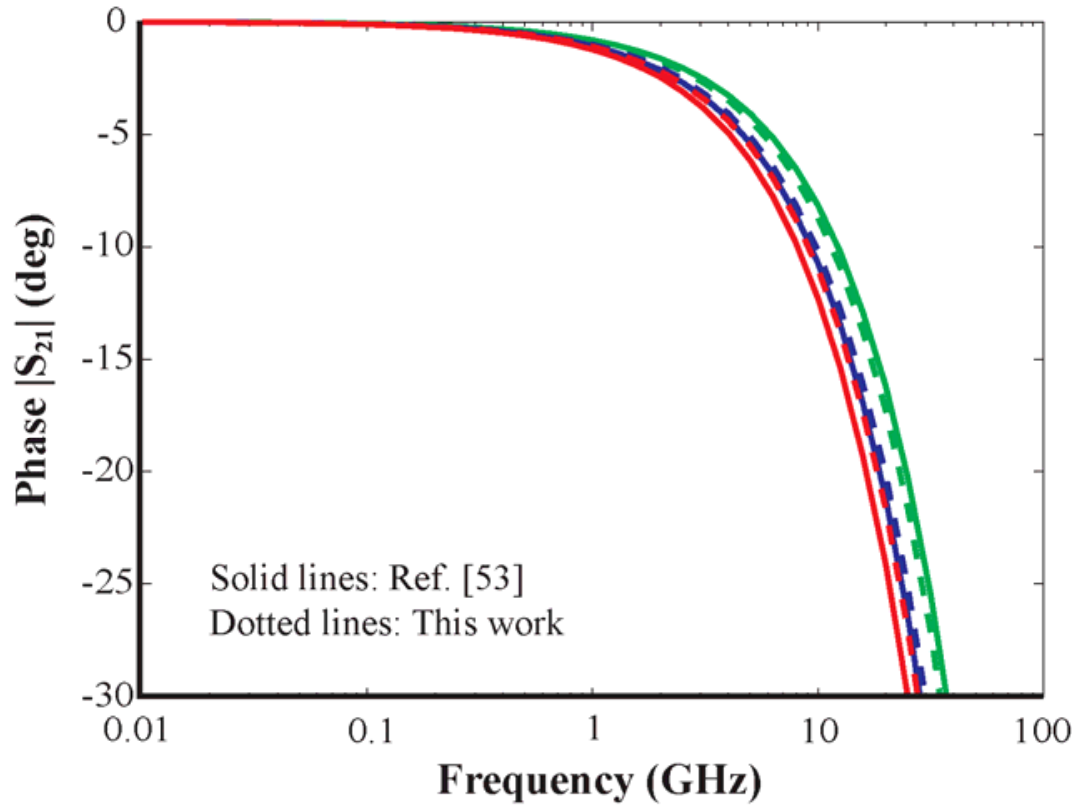

(b)

Fig. 11. Comparison of $\mathrm{S}_{21}$ from our model and Li et al. model [53] for MWCNT interconnects: (a) amplitude and (b) phase. 


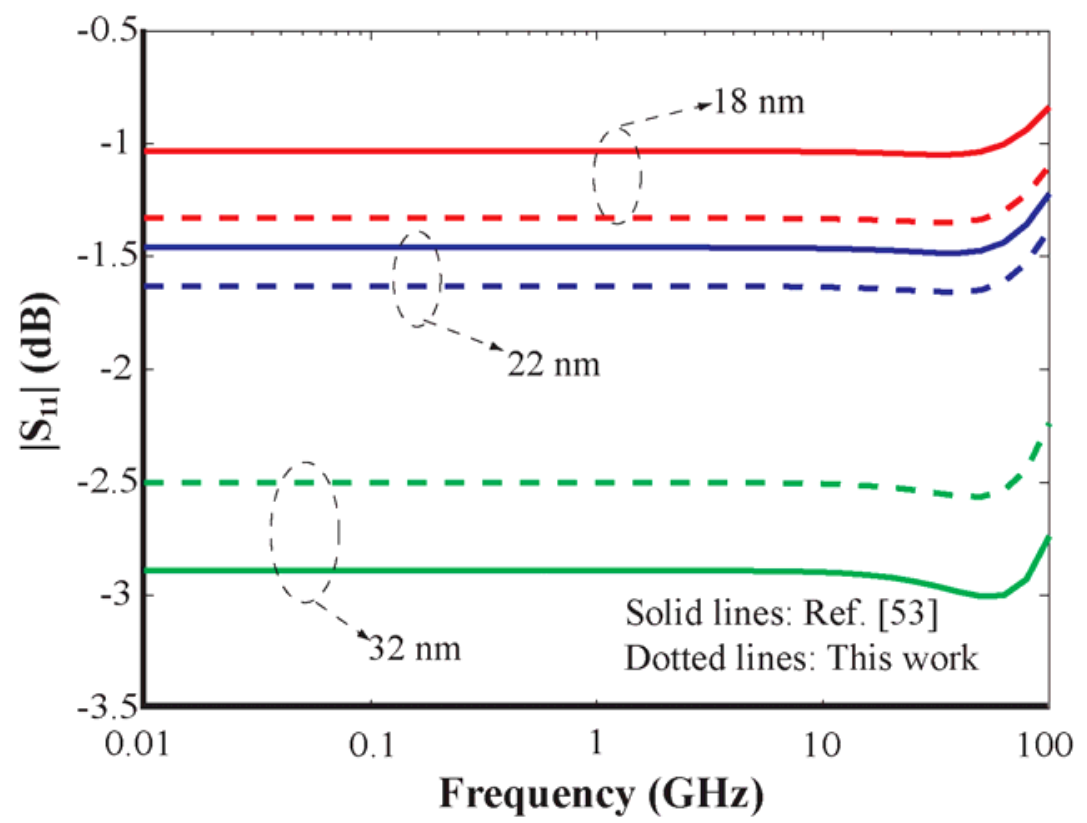

(a)

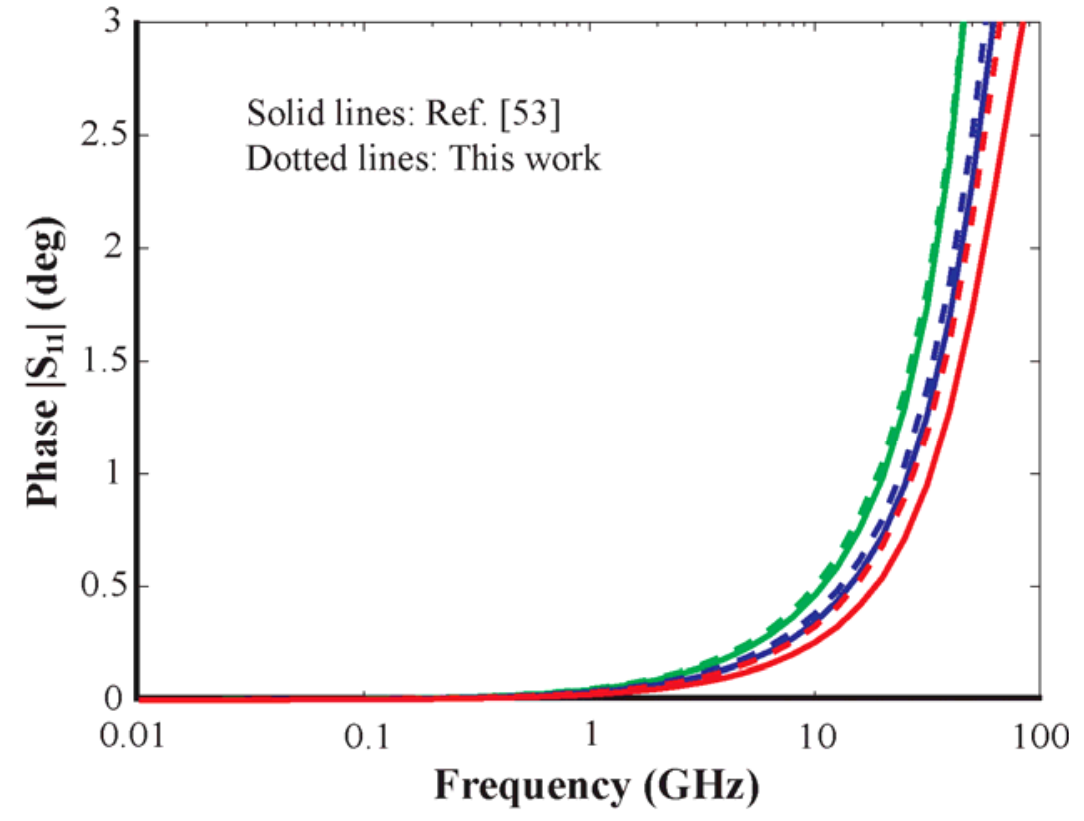

(b)

Fig. 12. Comparison of $\mathrm{S}_{11}$ from our model and Li et al. model [53] for MWCNT interconnects: (a) amplitude and (b) phase.

Figure 13 (a) and (b) show $\mathrm{S}_{21}$ and $\mathrm{S}_{11}$ parameters of MWCNT, SWCNT bundle and $\mathrm{Cu}$ interconnects of lengths corresponding to ballistic transport $(1 \mu \mathrm{m})$, local interconnection $(10$ $\mu \mathrm{m}$ and $100 \mu \mathrm{m})$ and global interconnection $(500 \mu \mathrm{m})$. For comparison, we choose $\beta=1 / 3$ and $50 \Omega$ terminal impedance, which is a typical impedance for high frequency transmission 
lines. For the MWCNT and SWCNT bundle, the electrostatic capacitance depends upon the geometry of the structure and is approximately equal to that of $\mathrm{Cu}$ interconnects $[12,53,83]$.

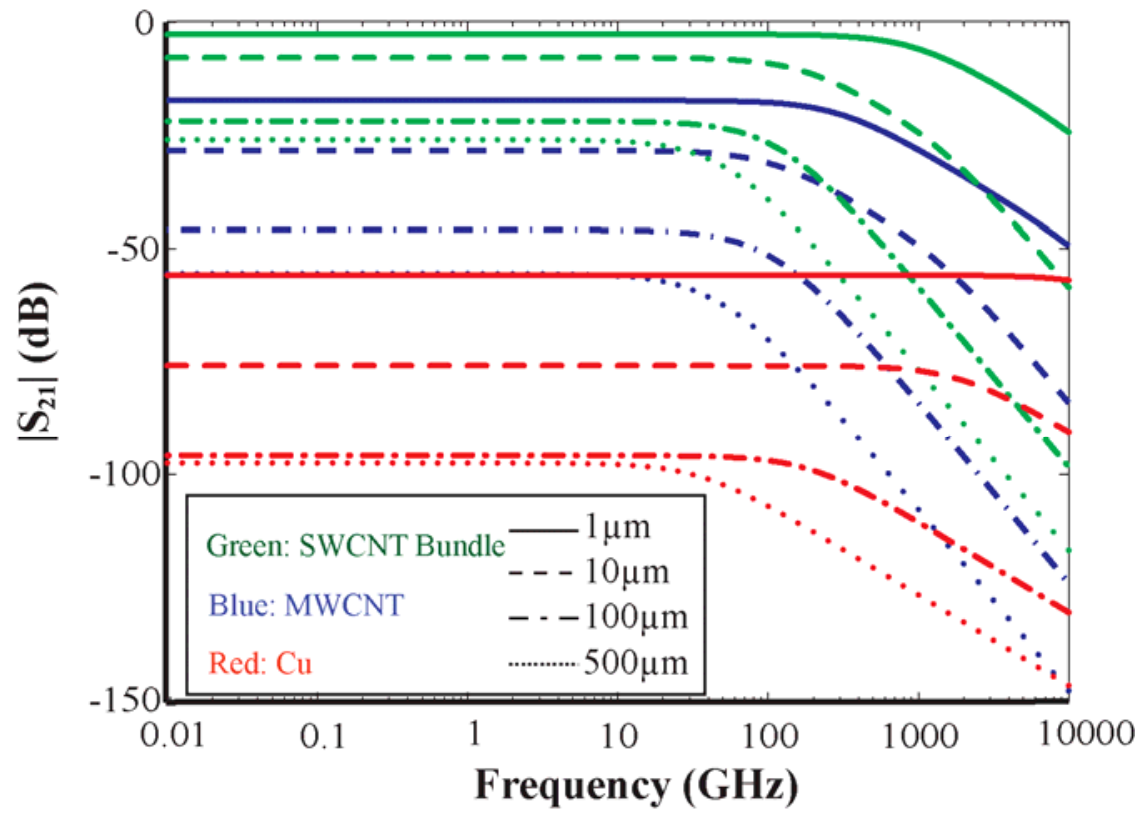

(a)

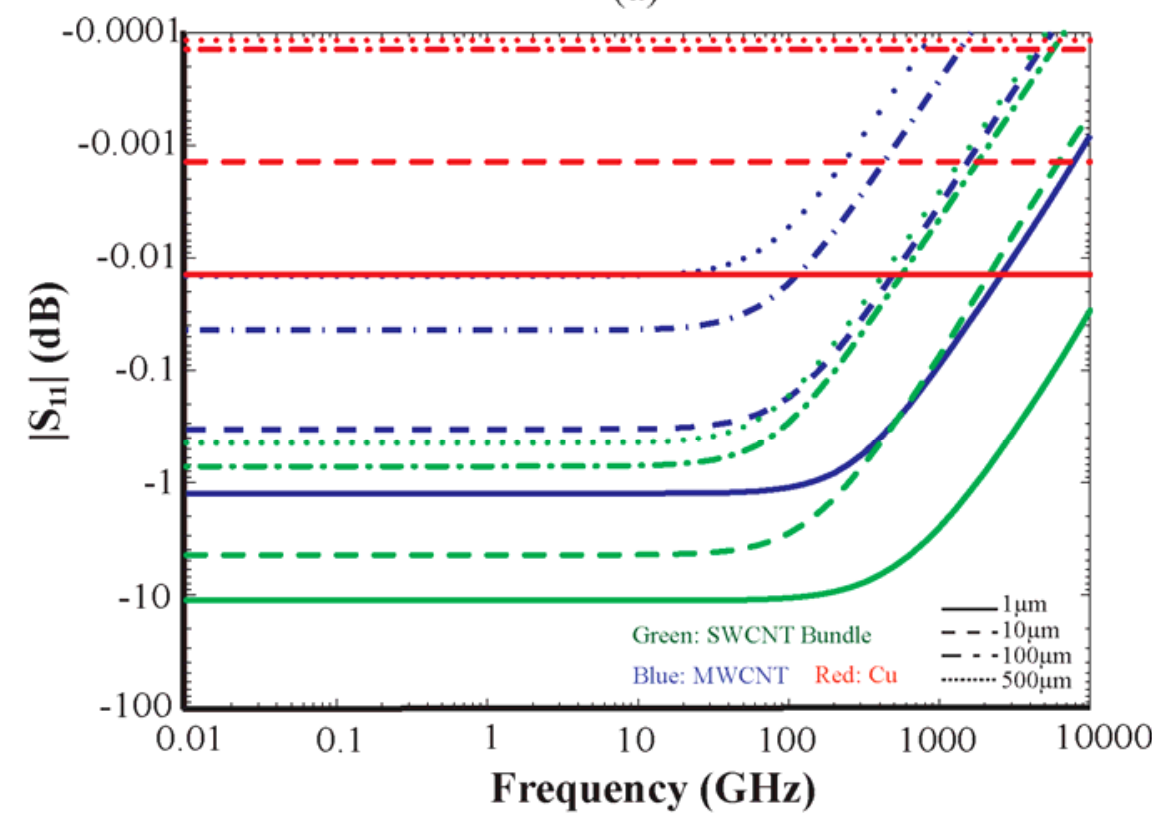

(b)

Fig. 13. Calculated S-parameters of different interconnects: (a) $\mathrm{S}_{21}$ (amplitude) and (b) $\mathrm{S}_{11}$ (amplitude).

Figure 13 (a) shows the $3 \mathrm{~dB}$ bandwidths for both the $\mathrm{CNT}$ and $\mathrm{Cu}$ interconnects. The transmission efficiency of both the CNT and $\mathrm{Cu}$ interconnects decreases with increasing lengths. However, $\mathrm{Cu}$ interconnect has a larger $3 \mathrm{~dB}$ bandwidth in comparison with $\mathrm{CNT}$ 
interconnects. This is because $\mathrm{Cu}$ has smaller inductance compared with CNT interconnects. It should also be noticed that the short length CNT interconnects still have over a $100 \mathrm{GHz}$ $3 \mathrm{~dB}$ bandwidth. Figure 13 (a) also shows large $\mathrm{S}_{21}$ for SWCNT bundle and MWCNT interconnects than that of the $\mathrm{Cu}$ interconnect. This is because SWCNT bundle and MWCNT have much smaller resistances. Furthermore SWCNT bundle has more connection channels than MWCNT; it has larger 3dB bandwidth and $\mathrm{S}_{21}$ value, which means larger transmission efficiency. In Fig. 13 (b) for $\mathrm{S}_{11}$ parameters at frequencies less than $100 \mathrm{GHz}, \mathrm{Cu}$ interconnect has the largest reflection losses while SWCNT bundle interconnect has the least reflection losses. The results show that SWCNT bundle interconnect has better performance than the MWCNT interconnect. This can be explained that the number of SWCNTs in the bundle is larger than that of shells in the MWCNT of the same size. It can be shown that for $22 \mathrm{~nm}$ width of SWCNT bundle and MWCNT interconnects, calculated number of SWCNTs in a bundle from $N=\beta(N x N y-[N y / 2])$ and the number of shells in a MWCNT from Eq. (11) are approximately 500 and 10 , respectively. It means that there are more conducting channels in the bundle according to one-dimensional fluid model.

Recently, Srivastava et al. [28] have obtained an analytical solution for the current transport in CNT-FETs for the analysis and design of CNT-FET based integrated circuits. Based on their work, a dynamic model [94] for CNT-FETs is obtained and Verilog-AMS language [95] is used to predict static and dynamic characteristics of CNT-FETs and integrated circuits. Verilog-AMS requires less computational steps and easy to experiment with the developing model equations. In this work, Verilog-AMS is used to describe CNTFET static and dynamic models and simulated CNT-FET circuits in Cadence/Spectre. Figure 14 shows the CNT-FET inverter pair at $l \mathrm{~V}$ supply voltage. The interconnection can be $\mathrm{Cu}$ or MWCNT or SWCNT bundle. The delay analysis includes the CNT-FET models developed by Srivastava et al. [28] and dynamic models reported in Ref. 94.

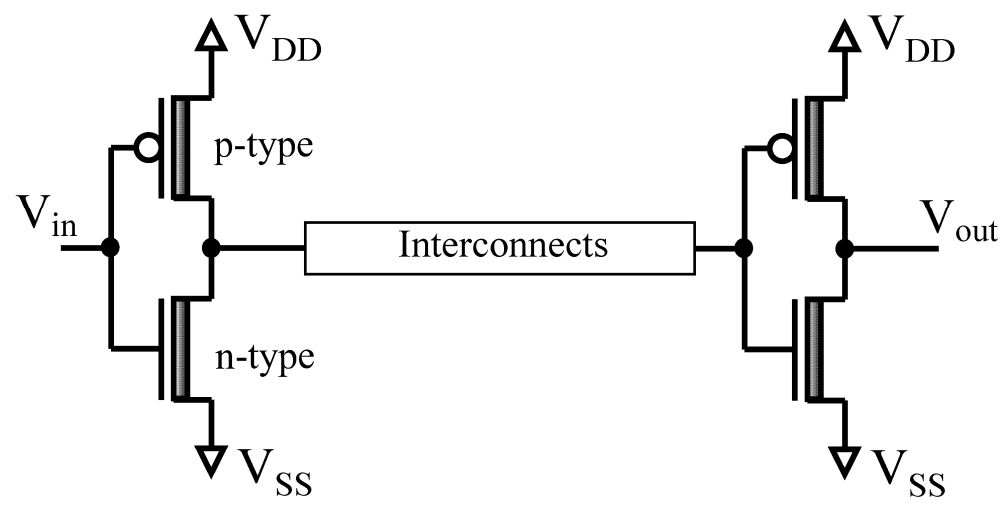

Fig. 14. Inverter pair with interconnects.

Figure 15 shows transient response of a CNT-FET inverter interconnected with $10 \mu \mathrm{m}$ long MWCNT and SWCNT bundle interconnection wires. Figure 15 also shows a comparison of transient response for an ideal interconnection (assuming direct connection) and $\mathrm{Cu}$ interconnection wire. Input signal is a $100 \mathrm{MHz}$ square pulse. The propagation delays of MWCNT interconnects $(\beta=1$ and $\beta=1 / 3)$ are close to ideal interconnect and are smaller than SWCNT bundle and $\mathrm{Cu}$ interconnects. As mentioned earlier, the number of SWCNTs in the bundle is larger than the number of shells in the MWCNT. Therefore, the resistance is smaller for the SWCNT bundle interconnect than that of the MWCNT. However, the capacitance of the SWCNT bundle interconnect is much larger than that of MWCNT. As a result, the propagation delay of MWCNT is smaller than that of the SWCNT bundle. The propagation delays are smaller for $\beta=1$ than for $\beta=1 / 3$ for both MWCNT and SWCNT 
bundle interconnects. This can be explained by the fact that there are more interconnect channels when $\beta$ increases.

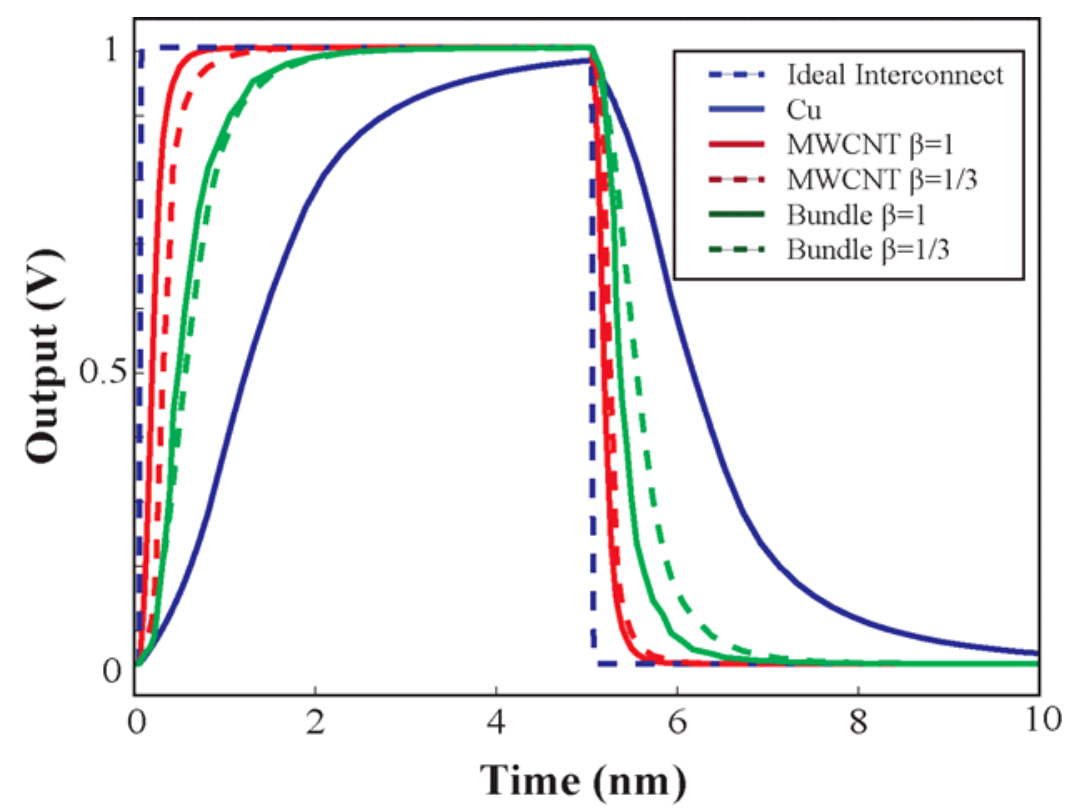

Fig. 15. Output waveforms of an inverter pair with $10 \mu \mathrm{m}$ length of different interconnect wires.

One of the advantages of CNT interconnect is its large mean free path, which is on the order of several microns as compared to $\sim 40 \mathrm{~nm}$ for $\mathrm{Cu}$ at room temperature. It provides low resistivity and possible ballistic transport in short-length interconnects [96]. In this work, we have also simulated a CNT-FET inverter pair with $1 \mu \mathrm{m} \mathrm{Cu}$, MWCNT and SWCNT bundle interconnects using Cadence/Spectre. Local interconnects are often used for connecting nearby gates or devices with lengths on the order of micrometers. Therefore, these have the smallest cross section and largest resistance per unit length compared to global interconnects. We have utilized the process parameters from the 2016 node for $22 \mathrm{~nm}$ technology [86] assuming $22 \mathrm{~nm}$ diameter of a MWCNT, $22 \mathrm{~nm}$ width and $44 \mathrm{~nm}$ thickness of a SWCNT bundle. Relatively global interconnects have larger cross section and smaller resistivity. The lengths are on the order of hundred micrometers. We have utilized the process parameters from the 2016 node of $22 \mathrm{~nm}$ technology [86] assuming $33 \mathrm{~nm}$ diameter of a MWCNT, $33 \mathrm{~nm}$ width and $87 \mathrm{~nm}$ thickness of a SWCNT bundle. Simulations are performed for different lengths of $\mathrm{Cu}$, MWCNT and SWCNT bundle interconnects corresponding to ballistic transport length $(1 \mu \mathrm{m})$, local interconnects $(10 \mu \mathrm{m}, 100 \mu \mathrm{m})$ to global interconnects $(500$ $\mu \mathrm{m})$. The results are shown in Fig. 16. Dependence of delay on interconnection length in Fig. 16 shows that the increase in delay for $\mathrm{Cu}$ interconnects is larger than that of MWCNT and SWCNT bundle interconnects. The delays of MWCNT interconnects $(\beta=1$ and $\beta=1 / 3)$ are smaller than that of SWCNT bundle and $\mathrm{Cu}$ interconnects. The delays are smaller for $\beta=1$ than for $\beta=1 / 3$ for both MWCNT and SWCNT bundle interconnects and is due to more interconnect channels with increase in $\beta$.

Power dissipation is another challenge to next generation interconnects. We have simulated power dissipation for MWCNT and SWCNT bundle interconnects in $22 \mathrm{~nm}$ technology node and compared with the $\mathrm{Cu}$ wire interconnects. Table 3 summarizes power dissipation ratio of MWCNT and SWCNT bundle $(\beta=1 / 3$ and $\beta=1)$ to $\mathrm{Cu}$ interconnect. CNT interconnects dissipates less power and especially for local interconnections. Maximum 
power dissipation in CNTs interconnections is no more than the $8 \%$ of the $\mathrm{Cu}$ interconnections.

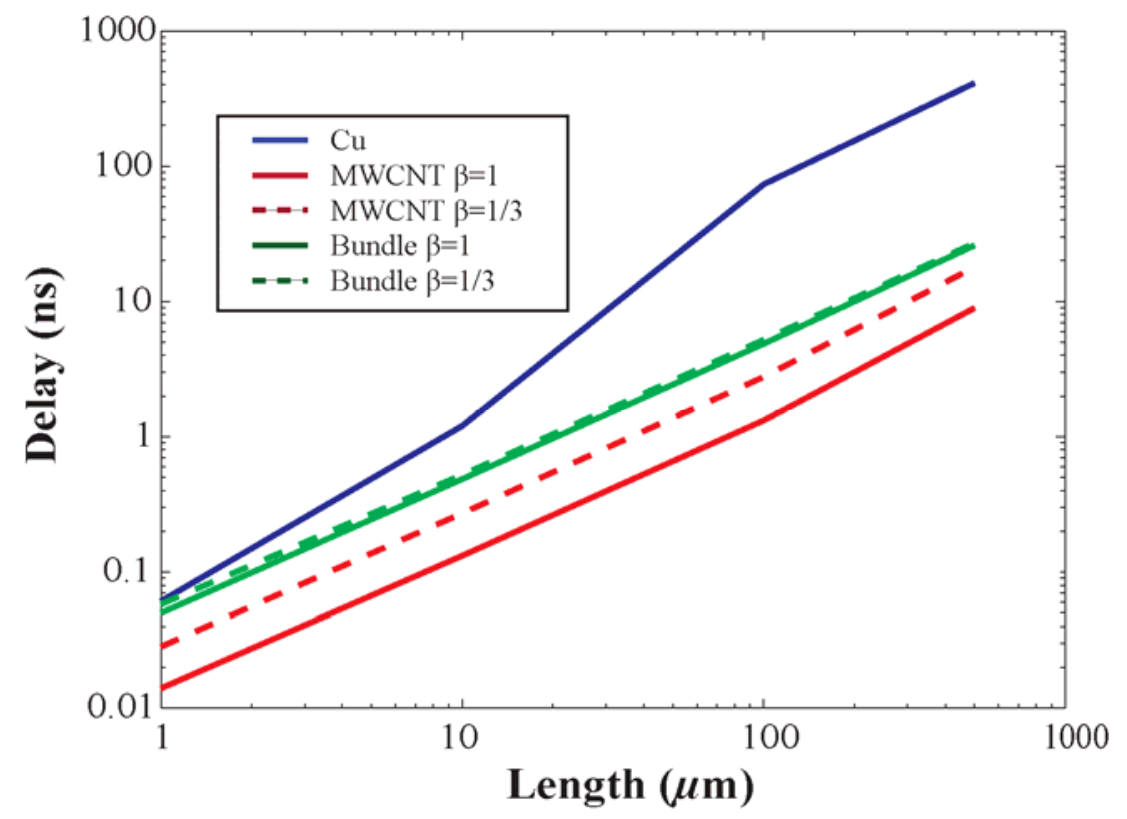

Fig. 16. Propagation delays of interconnects of different lengths for $22 \mathrm{~nm}$ technology.

Table 3: Power dissipation ratio of MWCNT and SWCNT bundle to $\mathrm{Cu}$ interconnects

\begin{tabular}{|l|c|c|c|c|}
\hline \multirow{2}{*}{ Type of CNT } & \multicolumn{4}{|c|}{ Normalized Power Dissipation (\%) } \\
\cline { 2 - 5 } & $\mathbf{1}$ & $\mathbf{1 0}$ & $\mathbf{1 0 0}$ & $\mathbf{5 0 0}$ \\
\cline { 2 - 5 } & 0.070 & 0.065 & 0.339 & 1.422 \\
\hline MWCNT $(\boldsymbol{\beta}=\mathbf{1})$ & 0.359 & 0.418 & 2.182 & 7.591 \\
\hline MWCNT $(\boldsymbol{\beta}=\mathbf{1 / 3})$ & 0.011 & 0.015 & 0.079 & 0.137 \\
\hline $\begin{array}{l}\text { SWCNT Bundle } \\
(\boldsymbol{\beta}=\mathbf{1})\end{array}$ & 0.036 & 0.047 & 0.256 & 0.688 \\
\hline $\begin{array}{l}\text { SWCNT Bundle } \\
(\boldsymbol{\beta}=\mathbf{1 / 3})\end{array}$ & & &
\end{tabular}

Note: Normalization parameter is the length of $\mathrm{Cu}(1,10,100$ and $500 \mu \mathrm{m})$. The technology node is $22 \mathrm{~nm}$.

\section{CONCLUSION}

In this paper, models for CNT interconnects, which include SWCNT, MWCNT and SWCNT bundle are discussed based on one-dimensional fluid theory. The one-dimensional fluid model can be applied to CNT interconnects using low resistance contacts in current low-voltage nanometer CMOS technologies. The applicability of MWCNT and SWCNT bundle as interconnect wires for next generation design of integrated circuits has been explored theoretically and compared with $\mathrm{Cu}$ interconnects in $22 \mathrm{~nm}$ technology node. Results of the one-dimensional fluid theory for SWCNT interconnect extended to MWCNT and SWCNT bundle interconnects show that MWCNT and SWCNT bundle interconnects have better performance than the $\mathrm{Cu}$ interconnects. MWCNT and SWCNT bundle interconnects exhibit 
higher transmission efficiency and lower reflection losses, smaller delays and less power dissipations. This is mainly due to larger conductivity of MWCNT and SWCNT bundle, proportional to the number of conducting shells $(M)$ in MWCNT and conducting SWCNTs $(N)$, respectively. With no special separation techniques, the metallic nanotubes are distributed with probability $\beta=1 / 3$. While the proportion of metallic nanotubes can be potentially increased using techniques introduced in Refs. 80 and 81, the delays in MWCNT and SWCNT bundle interconnects can be further decreased with increase in $\beta$ and approaching to 1 . It is also noticed that with the increase in interconnection length, the delay of $\mathrm{Cu}$ interconnect increases faster than that of MWCNT and SWCNT bundle interconnects. For applications requiring small circuit delays MWCNT interconnects should be used due to smaller capacitances. Applications requiring large transmission efficiency and low reflection losses, CNT bundles should be used for interconnects since the numbers of conducting channels per shell are more in SWCNTs bundle than the number of conducting channels per shell in MWCNT of the same size. These findings suggest that MWCNT and SWCNT bundle can replace $\mathrm{Cu}$ as interconnection wires in next generation of VLSI integrated circuits.

\section{Acknowledgment}

Part of the work is supported by the United States Air Force Research Laboratory under agreement number FA9453-10-1-0002. The U.S. Government is authorized to reproduce and distribute reprints for Government purposes notwithstanding any copyright notation thereon. In part the work is also supported under Contract \#NSF (2009)-PFUND-138. One of the authors, Yao $\mathrm{Xu}$ acknowledges the support provided by the Louisiana Economic Development Assistantship (EDA) program to carry out the proposed research.

\section{References}

[1] K.-H. Koo, P. Kapur, and K. C. Saraswat, "Compact performance models and comparison for gigascale on-chip global interconnect technologies," IEEE Trans. Electron Dev. 56, 1787-1798 (2009) [doi: 10.1109/TED.2009. 2026196].

[2] C. Ryu, K.-W. Kwon, A. L. S. Loke, H. Lee, T. Nogami, V. M. Dubin, R. A. Kavari, G. W. Ray, and S. S. Wong, "Microstructure and reliability of copper interconnects," IEEE Trans. Electron Dev. 46, 1113-1120 (1999) [doi: 10.1109/16.766872]

[3] D. A. B. Miller, "Optical interconnects to silicon," IEEE J. Sel. Top. Quantum Electron. 6, 1312-1317 (2000) [doi: 10.1109/2944. 902184].

[4] G. Chen, H. Chen, M. Haurylau, N. A. Nelson, D. H. Albonesi, P. M. Fauchet, and E. G. Friedman, "Predictions of CMOS compatible on-chip optical interconnect," Integrat. VLSI J. 40, 434-446 (2007) [doi: 10.1016/j.vlsi.2006. 10.001].

[5] P. Kapur and K. C. Saraswat, "Comparisons between electrical and optical interconnects for on-chip signaling," Proc. IEEE Int. Interconnect Technol. Conf., 89-91 (2002) [doi: 10.1109/IITC.2002.1014897].

[6] M. Haselman and S. Hauck, "The future of integrated circuits: a survey of nanoelectronics," Proc. IEEE 98, 11-38 (2010) [doi: 10.1109/ JPROC.2009.2032356].

[7] A. Maffucci, "Carbon nanotubes in nanopackaging applications," IEEE Nanotechnol. Mag. 3, 22-25 (2009) [doi: 10.1109/MNANO.2009. 934214].

[8] A. G. Chiariello, G. Miano, and A. Maffucci, "Carbon nanotube bundles as nanoscale chip to package interconnects," Proc. 9th IEEE Conf. Nanotechnol., 58-61 (2009). 
[9] J.-H. Ting, C.-C. Chiu, and F.-Y. Huang, "Carbon nanotube array vias for interconnect applications," J. Vac. Sci. Technol. B 27, 1086-1092 (2009) [doi: 10.1116/1.3123330].

[10] N. Alam, A. K. Kureshi, M. Hasan, and T. Arslan, "Carbon nanotube interconnects for low-power high-speed applications," Proc. IEEE Int. Symp. Circuits Syst., 22732276 (2009) [doi: 10.1109/ ISCAS.2009.5118252].

[11] M. R. Stan, D. Unluer, A. Ghosh, and F. Tseng, "Graphene devices, interconnect and circuits," Proc. IEEE Int. Symp. Circuits Syst., 69-72 (2009) [doi: 10.1109/ISCAS.2009.5117687].

[12] N. Srivastava, Li Hong, F. Kreupl, and K. Banerjee, "On the applicability of singlewalled carbon nanotubes as VLSI interconnects," IEEE Trans. Nanotechnol. 8, 542559 (2009) [doi: 10.1109/TNANO.2009.2013945].

[13] H. Li, C. Xu, N. Srivastava, and K. Banerjee, "Carbon nanomaterials for nextgeneration interconnects and passives: physics, status, and prospects," IEEE Trans. Electron. Dev. 56, 1799-1821 (2009) [doi: 10.1109/TED.2009.2026524].

[14] S. Iijima, "Helical microtubules of graphitic carbon," Nature 354, 56-58 (1991) [doi: 10.1038/354056a0].

[15] R. Saito, M. S. Dresselhaus, and G. Dresselhaus, Physical Properties of Carbon Nanotubes, Imperial College Press, London (1998).

[16] M. S. Dresselhaus, G. Dresselhaus, and P. Avouris, Carbon Nanotube: Synthesis, Properties, Structure, and Applications, Springer, Berlin(2001).

[17] P. Avouris, J. Appenzeller, R. Martel, and S. L. Wind, "Carbon nanotube electronics," Proc. IEEE 91, 1772-1784 (2003) [doi: 10.1109/JPROC. 2003.818338].

[18] T. S. Cho, K.-J. Lee, J. Kong, and A. P. Chandrakasan, "A low power carbon nanotube chemical sensor system," Proc. IEEE Custom Integrat. Circuits Conf., 181184 (2007).

[19] S. J. Tans, A. R. M. Vershueren, and C. Dekker, "Room-temperature transistor based on a single carbon nanotube," Nature 393, 49-52 (1998) [doi: 10.1038/29954].

[20] R. Martel, T. Schmidt, H. R. Shea, T. Hertel, and P. Avouris, "Single- and multi-wall carbon nanotube field effect transistors," Appl. Phys. Lett. 73, 2447-2449 (1998) [doi: 10.1063/1.122477].

[21] A. Bachtold, P. Hadley, T. Nakanishi, and C. Dekker, "Logic circuits with carbon nanotube transistors," Science 294, 1317-1320 (2001) [doi: 10.1126/science. 1065824].

[22] H. S. P. Wong, "Field effect transistors - from silicon MOSFETs to carbon nanotube FETs," Proc. $23^{\text {th }}$ Int. Conf. Microelectron., 103-107 (2002).

[23] A. Javey, Q. Wang, W. Kim, and H. Dai, "Advancements in complementary carbon nanotube field-effect transistors," IEDM Tech. Digest, $741-774$ (2003).

[24] A. Javey, Q. Wang, A. Ural, Y. Li, and H. Dai, "Carbon nanotube transistors arrays for multistage complementary logic and ring oscillators," Nano Lett. 2, 929-932 (2002) [doi: 10.1021/n1025647r].

[25] A. Raychowdhury, S. Mukhopadhyay, and K. Roy, "A circuit-compatible model of ballistic carbon nanotube field effect transistors," IEEE Trans. Comput. Aided Des. Integrat. Circuits Syst. 23, 1411-1420 (2004) [doi: 10.1109/TCAD.2004.835135].

[26] I. O'Connor, J. Liu, F. Gaffiot, F. Prégaldiny, C. Lallement, C. Maneux, J. Goguet, S. Frégonèse, T. Zimmer, L. Anghel, T.-T. Dang, and R. Leveugle, "CNTFET modeling and reconfigurable logic-circuit design," IEEE Trans. Circuits Syst. Part-1 54, 2365-2379 (2007) [doi: 10.1109/TCSI.2007.907835].

[27] S. Fregonese, C. Maneux, and T. Zimmer, "Implementation of tunneling phenomena in a CNTFET compact model," IEEE Trans. Electron. Dev. 56, 2224-2231 (2009) [doi: 10.1109/TED.2009.2028621]. 
[28] A. Srivastava, J. Marulanda, Y. Xu, and A. K. Sharma, "Current transport modeling of carbon nanotube field effect transistors," phys. status solidi (a) 206, 1569-1578 (2009) [doi: 10.1002/pssa.200824221].

[29] J. P. Novak, E. S. Snow, E. J. Houser, D. Park, J. L. Stepnowski, and R. A. McGill, "Nerve agent detection using networks of single-walled carbon nanotubes," Appl. Phys. Lett. 83, 4026-4028 (2003) [doi: 10.1063/1.1626265].

[30] E. S. Snow, F. K. Perkins, E. J. Houser, S. C. Badescu, and T. L. Reinecke, "Chemical detection with a single-walled carbon nanotube capacitor," Science 307, 1942-1945 (2005) [doi: 10.1126/science.1109128].

[31] P. Qi, O. Vermesh, M. Grecu, A. Javey, Q. Wang, H. Dai, S. Peng, and K. J. Cho, "Toward large arrays of multiplex functionalized carbon nanotube sensors for highly sensitive and selective molecular detection," Nano Lett. 3, 347-351 (2003) [doi: 10.1021/n1034010k].

[32] C. Staii, A. T. Johnson, M. Chen, and A. Gelperin, "DNA-decorated carbon nanotubes for chemical sensing," Nano Lett. 5, 1774-1778 (2005) [doi: 10.1021/n1051261f].

[33] A. Star, T. R. Han, V. Joshi, J. C. P. Gabriel, and G. Grüner, "Nanoelectronic carbon dioxide sensors," Adv. Mater. 16, 2049-2052 (2004) [doi: 10.1002/ adma.200400322].

[34] J. W. G. Wilder, L. C. Venema, A. G. Rinzler, R. E. Smalley, and C. Dekker, "Electronic structure of atomically resolved carbon nanotubes," Nature 391, 59-62 (1998) [doi: 10.1038/34139].

[35] T. Xu, Z. Wang, J. Miao, X. Chen, and C. M. Tan, "Aligned carbon nanotubes for through-wafer interconnects," Appl. Phys. Lett. 91, 042108 (2007) [doi: 10.1063/1.2759989].

[36] A. G. Chiariello, A. Maffucci, and G. Miano, "Signal integrity analysis of carbon nanotube on-chip interconnects," Proc. IEEE Work. Sign. Propagat. Intercon., 1-4 (2009) [doi: 10.1109/SPI.2009.5089842].

[37] J. J. Plombon, K.P. O'Brien, F. Gstrein, and V.M. Dubin, "High-frequency electrical properties of individual and bundled carbon nanotubes," Appl. Phys. Lett. 90, 063106 (2007) [doi: 10.1063/1.2437724].

[38] Z. Yao, C. L. Kane, and C. Dekker, "High-field electrical transport in single-wall carbon nanotubes," Physic. Rev. Lett. 84, 2941-2944 (2000) [doi: 10.1103/PhysRevLett.84.2941].

[39] L. Nougaret, G. Dambrine, S. Lepilliet, H. Happy, N. Chimot, V. Derycke, and J. P. Bourgoin, "Gigahertz characterization of a single carbon nanotube," Appl. Phys. Lett. 96, 042109 (2010) [doi: 10.1063/1.3284513].

[40] M. S. Sarto and A. Tamburrano, "Single-conductor transmission-line model of multiwall carbon nanotubes," IEEE Trans. Nanotechnol. 9, 82-92 (2010) [doi: 10.1109/TNANO.2009.2023516].

[41] K. S. Novoselov, A. K. Geim, S. V. Morozov, D. Jiang, Y. Zhang, S. V. Dubonos, I. V. Grigorieva, and A. A. Firso, "Electric field effect in atomically thin carbon films," Science 306, 666-669 (2004) [doi: 10.1126/science.1102896].

[42] A. Naeemi and J. D. Meindl, "Compact physics-based circuit models for graphene nanoribbon interconnects," IEEE Trans. Electron Dev. 56, 1822-1833 (2009) [doi: 10.1109/TED.2009.2026122].

[43] F. Kreupl, A. P. Graham, G. S. Duesberg, W. Steinhogl, M. Liebau, E. Unger, and W. Honlein, "Carbon nanotubes in interconnect applications," Microelectron. Eng. 64, 399-408 (2002) [doi: 10.1109/IEDM.2004.1419261].

[44] P. Patel-Predd, "Carbon-nanotube wiring gets real," IEEE Spectrum 14 (2008) http://spectrum.iee.org/semiconductors/materials/carbonnanotube-wiring-gets-real. 
[45] A. Raychowdhury and K. Roy, "Modeling and analysis of carbon nanotube interconnects and their effectiveness for high speed VLSI design," Proc. IEEE Conf. Nanotechnol., 608-610 (2004) [doi: 10.1109/NANO.2004. 1392435].

[46] N. Srivastava, and K. Banerjee, "Performance analysis of carbon nanotube interconnects for VLSI applications," IEEE/ACM Proc. Int. Conf. Comput. Aided Des., 383-390 (2005) [doi: 10.1109/ICCAD.2005.1560098].

[47] S. Salahuddin, M. Lundstrom, and S. Datta, "Transport effects on signal propagation in quantum wires," IEEE Trans. Electron. Dev. 52, 1734-1742 (2005) [doi: 10.1109/TED.2005.852170].

[48] A. Nieuwoudt and Y. Massoud, "Understanding the impact of inductance in carbon nanotubes for VLSI interconnect using scalable modeling techniques," IEEE Trans. Nanotechnol. 5, 758-765 (2006) [doi: 10.1109/TNANO. 2006.883480].

[49] H. Li and K. Banerjee, "High-frequency analysis of carbon nanotube interconnects and implications for on-chip inductor design," IEEE Trans. Electron. Dev. 56, 22022214 (2009) [doi: 10.1109/TED.2009.2028395].

[50] A. Nieuwoudt and Y. Massoud, "Evaluating the impact of resistance in carbon nanotube bundles for VLSI interconnect using diameter-dependent modeling technique," IEEE Trans. Electron. Dev. 53, 2460-2466 (2006) [doi: 10.1109/TED.2006.882035].

[51] A. Maffucci and G. Miano, "A new circuit model for carbon nanotube interconnects with diameter-dependent parameters," IEEE Trans. Nanotechnol. 8, 345-354 (2008) [doi: 10.1109/TNANO.2008.2010545].

[52] A. Naeemi and J. D. Meindl, "Compact physical models for multiwall carbonnanotube interconnects," IEEE Electron Dev. Lett. 27, 338-340 (2006) [doi: 10.1109/LED.2006.873765].

[53] H. Li, W.-Y. Yin, K. Banerjee, and J.-F. Mao, "Circuit modeling and performance analysis of multi-walled carbon nanotube interconnects," IEEE Trans Electron Dev. 55, 1328-1337 (2008) [doi: 10.1109/TED.2008.922855].

[54] D. Fathi, B. Forouzandeh, S. Mohajerzadeh, and R. Sarvari, "Accurate analysis of carbon nanotube interconnects using transmission line model," Micro Nano Lett. 4, 116-121 (2009) [doi: 10.1049/mnl.2009.0045].

[55] A. L. Fetter, "Electrodynamics of a layered electron gas. I. single layer," Ann. Phys. 81, 367-393 (1973) [doi: 10.1016/0003-4916(73)90161-9].

[56] A. L. Fetter, "Electrodynamics of a layered electron gas. II. periodic array," Ann. Phys. 88, 1-25 (1974) [doi: 10.1016/0003-4916(74)90397-2].

[57] A. Maffucci, G. Miano, and F. Villone, "A transmission line model for metallic carbon nanotube interconnects," Int. J. Circuit Theory Appl. 36, 31-51 (2008) [doi: 10.1002/cta.v36:1].

[58] C. Kane, L. Balents, and M. P. A. Fisher, "Coulomb interactions and mesoscopic effects in carbon nanotubes," Physical Rev. Lett. 79, 5086-5089 (1997) [doi: 10.1103/PhysRevLett.79.5086].

[59] M. Bockrath, D. H. Cobden, J. Lu, A. G. Rinzler, R.E. Smalley, L. Balents, and P. L. McEuen, "Lüttinger-Liquid behaviour in carbon nanotubes," Nature 397, 598-601 (1999) [doi: 10.1038/17569].

[60] H. Ishii, H. Kataura, H. Shiozawa, H. Yoshioka, H. Otsubo, Y. Takayama, T. Miyahara, S. Suzuki, Y. Achiba, M. Nakatake, T. Narimura, M. Higashiguchi, K. Shimada, H. Namatame, and M. Taniguchi, "Direct observation of TomonagaLüttinger-liquid state in carbon nanotubes at low temperatures," Nature 426, 540-544 (2003) [doi: 10.1038/nature02074].

[61] P. J. Burke, "An RF circuit model for carbon nanotubes," IEEE Trans. Nanotechnol. 2, 55-58 (2003) [doi: 10.1109/TNANO.2003.808503]. 
[62] P. J. Burke, "Lüttinger liquid theory as a model of the gigahertz electrical properties of carbon nanotubes," IEEE Trans. Nanotechnol. 1, 129-144 (2002) [doi: 10.1109/TNANO.2002.806823].

[63] M. P. A. Fisher and L. I. Glazman, "Transport in a one-dimensional Luttinger liquid," Mesoscopic Electron Transport, NATO-ASI Series E: Appl. Sci., pp. 331373, L. Kowenhoven, G. Schoen, and L. Sohn, Eds., Kluwer Academic Publishers, Dordrecht (1996).

[64] Y. Xu and A. Srivastava, "A model for carbon nanotube interconnects," Int. J. Circ. Theor. Appl., 1-17 (2009) [doi: 10.1002/ cta.587].

[65] G. Miano and F. Villone, "An integral formulation for the electrodynamics of metallic carbon nanotubes based on a fluid model," IEEE Trans. Antennas Propagat. 54, 2713-2724 (2006) [doi: 10.1109/TAP.2006.882170].

[66] A. G. Chiariello, A. Maffucci, G. Miano, F. Villone, and W. Zamboni, "Metallic carbon nanotube interconnects, part I: a fluid model and a 3D integral formulation," Proc. IEEE Work. Sign. Propagat. Interconnect., 181-184 (2006) [doi: 10.1109/SPI.2006.289215].

[67] G. K. Batchelor, An Introduction to Fluid Dynamics, Cambridge University Press (1967).

[68] J.-Y. Park, S. Rosenblatt, Y. Yaish, V. Sazonova, H. Ustunel, S. Braig, T. A. Arias, P. W. Brouwer, and P. L. McEuen, "Electron-phonon scattering in metallic singlewalled carbon nanotubes," Nano Lett. 4, 517-520 (2004) [doi: 10.1021/ nl035258c].

[69] A. Shadowitz, The Electromagnetic Field, Courier Dover Publications, Mineola, NY (1988).

[70] S. Ramo, J. R. Whinnery, and T. V. Duzer, Fields and Waves in Communication Electronics, Wiley, New York (1994).

[71] M. Nihei, D. Kondo, A. Kawabata, S. Sato, H. Shioya, M. Sakaue, T. Iwai, M. Ohfuti, and Y. Awano, "Low-resistance multi-walled carbon nanotube vias with parallel channel conduction of inner shells," Proc. IEEE Int. Interconnect Tech. Conf., pp. 234-236 (2005).

[72] H. J. Li, W. G. Lu, J. J. Li, X.D. Bai, and C.Z. Gu, "Multichannel ballistic transport in multiwall carbon nanotubes," Phys. Rev. Lett. 95, 86601 (2005) [doi: 10.1103/PhysRevLett.95.086601].

[73] Q. Yan, J. Wu, G. Zhou, W. Duan, and B.-L. Gu, "Ab initio study of transport properties of multiwalled carbon nanotubes," Physical Review B 72, 155425 (2005) [doi: 10.1103/PhysRevB.72.155425].

[74] L. Forró and C. Schönenberger, "Physical properties of multi-wall nanotubes," in Carbon Nanotubes: Synthesis, Structure, Properties and Applications, M. S. Dresselhaus, G. Dresselhaus and P. Avouris, Eds., Springer, Berlin(2000).

[75] S. Datta, Quantum Transport: Atom to Transistor, Cambridge University Press (2005).

[76] D. M. Rosenthal, and R. M. Asimow, Introduction to Properties of Materials, Van Nostrand Reinhold, New York (1971).

[77] Y. Xu, and A. Srivastava, "A model of multi-walled carbon nanotube interconnects," Proc. IEEE Int. $52^{\text {nd }}$ Midwest Symposium on Circuits and Systems (MWSCAS), 987990 (2009) [doi: 10.1109/MWSCAS.2009.5235934].

[78] A. Thess, R. Lee, P. Nikolaev, H. Dai, P. Petit, J. Robert, C. Xu, Y.H. Lee, S.G. Kim, A.G. Rinzler, D.T. Colbert, G.E. Scuseria, D. Tománek, J.E. Fischer, and R.E. Smalley, "Crystalline ropes of metallic carbon nanotubes," Science 273, 483-487 (1996) [doi: 10.1126/science.273.5274.483].

[79] M. Liebau, A.P. Graham, G.S. Duesberg, E. Unger, R. Seidel, and F. Kreupl, "Nanoelectronics based on carbon nanotubes: technological challenges and recent 
developments," Fullerene. Nanotube. Carbon Nanostruct. 13, 255-258 (2005) [doi: 10.1081/FST-200039292].

[80] N. Peng, Q. Zhang, J. Li, and N. Liu, "Influences of ac electric field on the spatial distribution of carbon nanotubes formed between electrodes," J. Appl. Phys. 100, 024309 (2006) [doi: 10.1063/1.2216476].

[81] M. Zheng, A. Jagota, M. S. Strano, A. P. Santos, P. Barone, S. G. Chou, B. A. Diner, M. S. Dresselhaus, R.S. McLean, G.B. Onoa, G. G. Samsonidze, E. D. Semke, M. Usrey, and D. J. Walls, "Structure-based carbon nanotube sorting by sequencedependent DNA assembly," Science 302, 1545-1548 (2003) [doi: 10.1126/ science.1091911].

[82] Y. Xu, A. Srivastava, and A. K. Sharma, "Emerging carbon nanotube electronic circuits, modeling and performance," VLSI Design 2010, 864165 (2009) [doi: $10.1155 / 2010 / 864165]$.

[83] A. Naeemi and J. D. Meindl, "Design and performance modeling for single-walled carbon nanotubes as local, semiglobal, and global interconnects in gigascale integrated systems," IEEE Trans. Electron. Dev. 54, 26-37 (2007) [doi: 10.1109/TED.2006.887210].

[84] A. Naeemi, J. A. Davis, and J. D. Meindl, "Compact physical models for multilevel interconnect crosstalk in gigascale integration (GSI)," IEEE Trans. Electron. Dev. 51, 1902-1912 (2004) [doi: 10.1109/TED.2004.837379].

[85] G. Ya. Slepyan, N. A. Krapivin, S. A. Maksimenko, A. Lakhtakia, and O. M. Yevtushenko, "Scattering of electromagnetic waves by a semi-infinite carbon nanotube," Int. J. Electron. Commun. 55, 273-280 (2001) [doi: 10.1078/ 1434-841100041].

[86] International Technology Roadmap for Semiconductors (http://www.itrs.net/Links/ 2007ITRS/Home2007.htm).

[87] C. P. Yue and S. S. Wong, "Physical modeling of spiral inductors on silicon," IEEE Trans. Electron. Dev. 47, 560-568 (2000) [doi: 10.1109/16.824729].

[88] Y. Massoud and A. Nieuwoudt, "Modeling and design challenges and solutions for carbon nanotube-based interconnect in future high performance integrated circuits," J. Emerg. Technol. Comput. Syst. 2, 155-196 (2006) [doi: 10.1145/1167943. 1167944].

[89] A. Nieuwoudt and Y. Massoud, "On the optimal design, performance, and reliability of future carbon nanotube-based interconnect solutions," IEEE Trans. Electron. Dev. 55, 2097-2110 (2008) [doi: 10.1109/TED.2008.926733].

[90] G. F. Close and H. S. P. Wong, "Fabrication and characterization of carbon nanotube interconnects," IEDM Technol. Digest, 203-206 (2007) [doi: 10.1109/IEDM.2007. 4418902].

[91] G. F. Close, S. Yasuda, B. Paul, S. Fujita, and H.-S. Philip Wong, "A $1 \mathrm{GHz}$ integrated circuit with carbon nanotube interconnects and silicon transistors," Nano Lett. 8, 706-709 (2008) [doi:10.1021/n10730965].

[92] G. F. Close, S. Yasuda, B. C. Paul, S. Fujita, and H.-S. P. Wong, "Measurment of subnanosecond delay through multiwall carbon-nanotube local interconnects in a CMOS integrated circuit," IEEE Trans. Electron. Dev. 56, 43-49 (2009) [doi: 10.1109/TED.2008.2008682].

[93] G. Ya. Slepyan, S. A. Maksimenko, A. Lakhtakia, O. Yevtushenko, and A.V. Gusakov, "Electrodynamics of carbon nanotubes: dynamic conductivity, impedance boundary conditions, and surface wave propagation," Physic. Rev. B 60, 1713617149 (1999) [doi: 10.1103/PhysRevB.60.17136].

[94] Y. Xu and A. Srivastava, "Dynamic response of carbon nanotube field effect transistor circuits," Proc. 2009 NSTI Nanotechnol. Conf. Expo. 1, 625-628 (2009). 
[95] Verilog-AMS Language Reference Manual (http://www.designers-guide.org/ VerilogAMS/).

[96] P. L. McEuen, M. S. Fuhrer, and H. Park, "Single-walled carbon nanotube electronics," IEEE Trans. Nanotechnol. 1, 78-85 (2002) [doi: 10.1109/TNANO.2002.1005429].

Ashok Srivastava is a professor in the Department of Electrical and Computer Engineering, Louisiana State University, Baton Rouge. He received Ph.D. in the field of Semiconductor Electronics, M. Tech. in Solid State Physics from the Indian Institute of Technology, Delhi, India in 1975, 1970, respectively and M.Sc. in Physics (Advanced Electronics) from the University of Lucknow, India in 1968. He is a senior member of IEEE, Electron Devices, Circuits and Systems, and Solid-State Circuits Societies, and member of SPIE and ASEE. His current research interests include Nanoelectronics (Quantum Electronic Devices, Carbon Nanotube - Interconnects, CNT-FETs and Integrated Circuits); Low Power VLSI Circuit Design, Testability and Noise Analysis (Digital, Analog and Mixed-Signal); Micro/NanoSystems; Radiation-hardened Integrated Circuits; and Low-Temperature Electronics. URL: http://www.ece.lsu.edu/ashok/

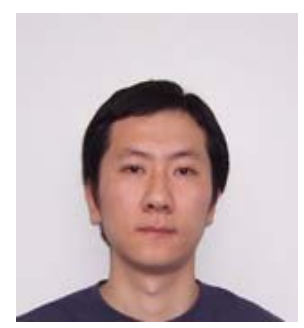

Yao Xu is a $\mathrm{PhD}$ student in Department of Electrical and Computer Engineering at the Louisiana State University. He received his BS and MS degrees in Photo Electronics from Tsinghua University and Chinese Academy of Sciences in 2000 and 2003, respectively, and his MS degree in Electrical Engineering from Louisiana State University in 2008. His current research interests include Nanoelectronics (Quantum Electronic Devices, Carbon Nanotube (CNT) Interconnects, CNT-FETs and Integrated Circuits), and Low Power VLSI Circuit Design.

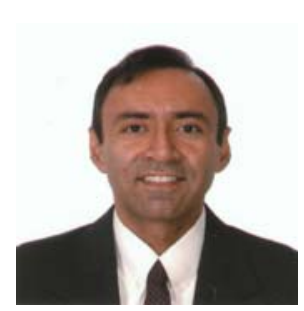

Ashwani K. Sharma received the B.S., M.S. and Ph.D. in electrical engineering in 1990, 1994 and 2004, respectively, from the University of New Mexico. Since 1994, he has been at the Air Force Research Laboratory at Kirtland AFB NM. His research interests include physics of nanostructures, nanoscale semiconductor device fabrication techniques, and nanodevices for high-speed and low-powerconsumption electronic/optoelectronic applications. He also holds a position as a research faculty at the University of New Mexico with the department of electrical engineering since 2006. 